(Invited Review for Special Issue: Plant Polyphenol)

\title{
The Flavonoid Biosynthetic Pathway in Arabidopsis: Structural and Genetic Diversity
}

Kazuki Saito ${ }^{1,2}$, Keiko Yonekura-Sakakibara ${ }^{1}$, Ryo Nakabayashi ${ }^{1}$, Yasuhiro Higashi ${ }^{1}$, Mami Yamazaki ${ }^{2}$, Takayuki Tohge ${ }^{3}$ and Alisdair R. Fernie ${ }^{3}$

${ }^{1}$ RIKEN Plant Science Center, 1-7-22 Suehiro-cho, Tsurumi-ku, Yokohama, 230-0045, Japan

${ }^{2}$ Graduate School of Pharmaceutical Sciences, Chiba University, Inohana 1-8-1, Chiba, 260-8675, Japan

${ }^{3}$ Max Planck Institute for Molecular Plant Physiology, Golm, Germany

Corresponding author:

Kazuki Saito

RIKEN Plant Science Center, 1-7-22 Suehiro-cho, Tsurumi-ku, Yokohama, 230-0045, Japan

Tel: $+81-45-503-9488$

Fax: $+81-45-503-9489$

e-mail: ksaito@psc.riken.jp 


\begin{abstract}
Flavonoids are representative plant secondary products. In the model plant Arabidopsis thaliana, at least 54 flavonoid molecules (35 flavonols, 11 anthocyanins and 8 proanthocyanidins) are found. Scaffold structures of flavonoids in Arabidopsis are relatively simple. These include kaempferol, quercetin and isorhamnetin for flavonols, cyanidin for anthocyanins and epicatechin for proanthocyanidins. The chemical diversity of flavonoids increases enormously by tailoring reactions which modify these scaffolds, including glycosylation, methylation and acylation. Genes responsible for the formation of flavonoid aglycone structures and their subsequent modification reactions have been extensively characterized by functional genomic efforts - mostly the integration of transcriptomics and metabolic profiling followed by reverse genetic experimentation. This review describes the state-of-art of flavonoid biosynthetic pathway in Arabidopsis regarding both structural and genetic diversity, focusing on the genes encoding enzymes for the biosynthetic reactions and vacuole translocation.
\end{abstract}




\section{Introduction}

Flavonoids are extensively distributed in the plant kingdom and form a group of representative plant secondary products. Given the ubiquitous distribution of flavonoids, they stride the boundary of primary and secondary metabolism. Since these compounds display several physiological functions in response to environmental factors, notwithstanding the fact that they do not play an essential role in plant growth, flavonoid biosynthetic genes have evolved to become one of the most widely-spread pathways in plants. Interestingly, this is also reflected in quantitative terms with the total carbon flux through the pathway constitutes approximately $20 \%$ of the total carbon flux [1]. As often claimed, flavonoids exhibit a variety of biological activities not only to plants, which produce these compounds, but also to animals, which intake flavonoids in their diet [2-4]. In the model plant Arabidopsis thaliana, flavonoids are one of major secondary products. The total number of metabolites (metabolome) in A. thaliana is estimated to exceed $5,000[5,6]$. Linking metabolome to genome is a challenging issue even in A. thaliana, in which the genomic resources far outstrip those of other plant species. However, investigations of flavonoid associated gene annotation have proven

highly successful in Arabidopsis. In this review, we discuss the structural diversity of flavonoid molecules and their biosynthetic genes encoding enzymes for the reactions and vacuole translocation but not about transcription factors, in order to understand the entire genetic basis of biosynthesis of this class of plant natural products. Since this review does not describe all aspects of biosynthesis of flavonoids in plant species besides $A$. thaliana, we refer readers to the recent more comprehensive articles on genetics and biochemistry of plant flavonoid biosynthesis [7-10].

\section{Flavonoid molecules and their differential accumulation in Arabidopsis}

In general, flavonoids are sub-classified into several families including flavonol, flavone, flavanone, flavan-3-ol, isoflavone and anthocyanidin according to the structure of and the modifications to the $\mathrm{A}, \mathrm{B}$ and $\mathrm{C}$ rings which constitute their structural chemical. In $A$. thaliana, several flavonol- and anthocyanidin-derivatives have additionally been isolated and characterized. Given that $A$. thaliana is the premier model plant for post-genomic biology flavonoid profiling in this species has greatly advanced since the introduction and application of liquid chromatography-mass spectrometry (LC-MS) approaches. Using such approaches renders the elucidation of key physicochemical properties derived from flavonoid structures considerably more facile 
than previously [11-13]. Flavonols and anthocyanins are identifiable since they display clear spectral absorbance of wavelengths at around $340 \mathrm{~nm}$ and $520 \mathrm{~nm}$ due to the presence of flavonol and modified flavilium aglycone (glycosyl and acyl adducts), respectively. Similarly, proanthocyanidins are identifiable since they display absorbance at 280/320 $\mathrm{nm}$ due to the presence of more highly condensed flavan-3-ol units. All of these metabolite groups have ether, ester and C4-C8 bonds which are easily cleaved in $\mathrm{MS} / \mathrm{MS}$ analysis. Subsequently to these analyses deciphering the MS/MS fragmentation patterns obtained using public MS and MS/MS resources from databases and literatures [14-16] readily supports chemical assignment of aglycone structures as well as other modifications. Such LC-MS-based flavonoid profiling has allowed chemical structural assignments as well as a metabolite abundance atlas of flavonoids in A. thaliana as shown in Figure 1. A. thaliana has been demonstrated to accumulate at least 54 flavonoid molecules (breaking down as 35 flavonols, 11 anthocyanins and eight proanthocyanidins). Some structures have been unequivocally identified by isolation and subsequent structural analysis by MS and NMR studies, whilst others were, thus far, only annotated or characterized by UV and/or MS/MS analysis with the comparison of the public data from known flavonoids [14, 15, 17-29].

Although accumulation of anthocyanin, which presents a clear visible phenotypic change in various tissues, has been used as a stress marker metabolite in many publications, details concerning the exact chemical structure of the anthocyanins present are reported in relatively few publications. Major anthocyanin structures in $A$. thaliana have firstly been determined by Bloor and Abrahams [19]. Subsequently, other major intermediates of anthocyanin metabolism were isolated and identified following LC-MS profiling of PAP1 (Production of anthocyanin pigment 1, AtMYB75, At1g56650) overexpressing Arabidopsis lines [20] and wild-type Arabidopsis grown under a diverse set of severe environmental challenges [30]. Many of these intermediates were subsequently purified (nomosts and unequivocally characterized with respect to their structures and relative stereochemistry [26]. The compound named as A11 (cyanidin-3-O-[2-O-(2-O-(sinapoyl)- $\beta$-D-xylosyl) -6- $O$-(4-O-( $\beta$-D-glucosyl)- $p$-coumaroyl- $\beta$-D-glucoside $] \quad$-5- $O$-[6- $O$-(malonyl) $\beta$-D-glucoside]) is the most highly modified cyanidin type anthocyanin with 3 acylated and 4 glycosylated parts. The compounds, A1 - A10, are presumed to be intermediates or less modified compounds of A11.

Flavonol glycosides in $A$. thaliana consist of the combination of aglycone (kaempferol, quercetin and isorhamnetin), glycosylation (mono, di and tri), and sugar (glucose, rhamnose and arabinose) groups. The abundance of these flavonoid molecules 
follows strict spatial regulation $[24,25]$. Relatively simple kaempferol and quercetin di-glycosides (di-rhamnosides or glucoside/rhamnoside in -3-O- and -7-O-positions) and tri-glycosides (di-rhamnosides and mono-glucoside in -3-O-, -2"-O- and -7-Opositions) are commonly distributed in all tissues of wild-type Arabidopsis. In the roots grown on the MS agar plates, four unique flavonol conjugates which are absent in every other tissue, including Arabidopsis roots grown in soil, were detected [24]. On the other hand, flavonols exhibiting unique arabinosylation modifications specifically accumulate in flowers. Furthermore, two flavonol-3-O-glucoside-hexosides were specifically found in flowers [25]. Interestingly, a triple knock mutant of Arabidopsis flavonol regulators PFG1, PFG2 and PFG3, previously named AtMYB12 (At2g47460), AtMYB11 (At3g62610) and AtMYB111 (At5g49330), displayed accumulation of these flavonol glycosides in pollen [31]. In recent metabolomics study, MS/MS spectral tag-based annotation profile additionally revealed the presence of malonylated flavonols in Arabidopsis leaves (kaempferol/quercetin-hexose-rhamnoside-malonyl) in inflorescences [15]. In seed of Arabidopsis, quercetin-3-O-rhamnoside, biflavonols (dimers of quercetin-rhamnoside), epicatechin and procyanidins accumulated in the seed coat, whereas diglycosylated flavonols were essentially detected in the embryo [22].

Proanthocyanidins or condensed tannins specifically accumulated in seed coat along with the maturation of seed conferring a brown hue [10, 22, 32]. In Arabidopsis these proanthocyanidins are formed using epicatechin as the basic condensing unit at C4-C8 positions. Catechin, which is a stereoisomer of epicatechin, is also known to be one of the building blocks of proanthocyanidins in other species [33-35], but as yet catechin-based proanthocyanidins have not been found in Arabidopsis.

\section{Flavonoid diversification in Arabidopsis natural variants}

A diverse array of flavonoids in higher plants have evolved according to their wide variety of physiological and ecological functions, which are required to meet adapting and conflicting demands to various environmental pressures under which plant communities grow. Illustrative but by no means comprehensive examples include high-light/UV-stress, cold stress, nutritional deficiencies and pathogen protection wherein the structure: function nexus has been the subject of considerable research [36-41]. Several studies have been made on natural variation of Arabidopsis accessions, in the case of flavonoid biosynthesis, elucidation of qualitative and quantitative differences between accessions have been performed and discussed in combination with stress treatments. Since there are numerous examples to illustrate biological and 
ecological importance of anthocyanins in plants, their variations have been much investigated [42-44]. Intriguingly, since the major anthocyanin A11 was isolated in Col-7 [19], it has been found in several other accessions including Col-0 [20, 26] and C24, Can, Co, Cvi, Ler, Rsch and Te [45] as a major anthocyanin. However, to date, only limited information is available with respect to quantitative and qualitative differences such as chemical variation of Arabidopsis flavonols and anthocyanins [46]. However, in general the pathways of flavonoid biosynthesis exhibit large variation in their aglycone backbones and tailoring modification which is likely explainable by functional divergence of key genes such as cytochrome P450s (CYPs), 2-oxoglutarate-dependent dioxygenases (2-ODDs) and UDP-glycosyltransferases (UGTs) during the evolution [38, 47-52]. That said natural variance of Arabidopsis anthocyanin levels have been studied particularly with respect to elucidating the functional relationships between the accumulation of anthocyanin and nitrogen limitation [43], cold acclimation [45] or high carbon stress [42].

Despite displaying less variability in chemical diversity than Arabidopsis anthocyanins, accessions-specific flavonol glycosides have been reported in leaves of several accessions, for example, kaempferol 3-O- $\beta$-glucosyl-(6-O- $\beta$-glucoside)-7-O- $\alpha$-rhamnoside $\quad$ in $\quad \mathrm{Ws}-0 \quad[18]$; quercetin/kaempferol/isorhamnetin-deoxyhexosyl-dihexoside in Ler [53]; kaempferol 3-O-rhamnosyl-glucosyl-glucoside-7-O-rhamnoside in $\mathrm{C} 24$ [23] and kaempferol-hexosyl-hexoside-deoxyhexoside in Ms-0 [54]. Moreover quantitative differences of seed specific biflavonols was observed with higher accumulation in Oy-0, Cvi-0, Bur-0 and St-0 [55]. Whilst accession-specific genes involved in Arabidopsis flavonoid biosynthesis have not yet been found, their elucidation and characterization will likely be critically important for enhancing our understanding plant evolution from the perspective of environmental adaptation.

\section{Genes involved in flavonoid biosynthesis}

\subsection{Shikimate pathway}

Flavonoids are synthesized by the combination of the phenylpropanoid and polyketide pathways (Figure 2). The phenylpropanoid pathway provides $p$-coumaroyl-CoA. The polyketide pathway is responsible for $\mathrm{C} 2$ chain elongation by utilizing malonyl-CoA as the condensing unit. The phenylpropanoid pathway initiates from the aromatic amino acids phenylalanine and tyrosine, which are synthesized by the 
shikimate pathway. The genes involved in the shikimate pathway in A. thaliana have been extensively documented in recent literature [56, 57].

4.2. Prior to the branch-point between flavonoids and general phenylpropanoids

Phenylalanine ammonia-lyase (PAL) - Phenylalanine ammonia-lyase (PAL) is the first committed enzyme in the phenylpropanoid pathway leading to the production of lignins, lignans and flavonoids. This enzyme catalyzes the formation of trans-cinnamic acid from phenylalanine with the concomitant loss of ammonia. In $A$. thaliana, a small gene family comprising four genes designated AtPAL1 - AtPAL4 (AtPAL1, At2g37040; AtPAL2, At3g53260; AtPAL3, At5g04230; AtPAL4, At3g10340) encode the isoforms of PAL $[58,59]$. They exhibit differential expression patterns and partially complement the function of each other in respective mutation [56, 60-62]. However, AtPAL1 and AtPAL2 are presumed to represent the principal isoforms responsible for the formation of phenylpropanoids in $A$. thaliana [56, 61]. In fact, pal1 pal2 double mutants exhibited a reduced production of anthocyanins and proanthocyanidins [63].

Cinnamic acid 4-hydroxylase $(\mathrm{C} 4 \mathrm{H})$ - Cinnamic acid 4-hydroxylase $(\mathrm{C} 4 \mathrm{H})$ is a cytochrome $\mathrm{P} 450$ monooxygenase which catalyzes the hydroxylation of trans-cinnamic acid at $\mathrm{C}-4$ position to yield $p$-coumaric acid (4-coumaric acid). In Arabidopsis, there is only one isoform in the subfamily of CYP73 designated CYP73A5 (AtC4H, REF3, At2g30490) [64, 65]. Seeds of plants exhibiting a mutation in this gene (ref3) exhibited reduced levels of proanthocyanidins in addition to reduced accumulation of wild-type levels of sinapoyl-malate and lignins [66].

4-Coumaric acid:CoA ligase (4CL) - $p$-Coumaric acid needs to be activated to the corresponding $\mathrm{CoA}$ thioester to facilitate further conversion. This reaction is catalyzed by 4-coumaric acid:CoA ligase (4CL) with the consumption of ATP as an energy source. Four isoforms of 4CL, At4CL1 - At4CL4 (At4CL1, At1g51680; At4CL2, At3g21240; At4CL3, At1g65060; At4CL5 (At4CL4), At3g21230), are present in the $A$. thaliana genome. From the results of expression patterns and phylogenetic similarity, At4CL3 is suggested to play a role in flavonoid biosynthesis, while At4CL1 and At4CL2 are presumed to be involved in lignin production [67]. At4CL5 (At4CL4) exhibits a substrate preference to ferulic acid and sinapic acid over $p$-coumaric acid, suggesting a different function from that of other 4CLs in Arabidopsis [68].

\subsection{Formation of malonyl-CoA}


Acetyl-CoA carboxylase (ACC) is an $\mathrm{Mg}^{2+}$-ATP dependent biotinylated protein complex that catalyzes the carboxylation of acetyl-CoA into malonyl-CoA [69-71]. The reaction takes place in two steps. First, bicarbonate as the source of carbon is transferred to the biotin molecule covalently bound to ACC (biotin carboxylase). This carbon-carbon bond formation requires energy derived from the hydrolysis of ATP. The second step is the transfer of the carboxyl group to acetyl-CoA to form malonyl-CoA (carboxyltransferase).

Arabidopsis has three ACC isozymes. A heteromeric form of ACC is composed of four distinct subunits: a biotin carboxyl carrier protein (BCCP1, CAC1A, At5g16390), biotin carboxylase (BC, CAC2, At5g35360), carboxyltransferase $\alpha$ subunit (CAC3, At2g38040) and $\beta$ subunit (accD, AtCg00500) [72]. These subunits form a protein complex in plastids and catalyze the first step reaction in de novo fatty acid biosynthesis. A homomeric form of ACC is composed of a large polypeptide with four functional domains. The Arabidopsis genome contains two genes encoding the homomeric type proteins located in a tandem repeat within a 25-kbp region (ACC1, At1g36160; ACC2, Atlg36180) [73, 74]. ACC1 is the cytosolic isozyme in Arabidopsis and produces malonyl-CoA for flavonoid biosynthesis. ACC2 has a longer $\mathrm{N}$-terminal sequence, and GFP-fusion protein experiments suggest that this confers a chloroplastic localisation [75]. ACC2 is likely functionally redundant in plastidic malonyl-CoA biosynthesis during seedling development. Biotinylation of ACC is catalyzed by a dual targeting of holocarboxylase synthetase 1 (HCS1, At2g25710) [76]. Cytosolic acetyl-CoA is synthesized by $\alpha_{4} \beta_{4}$ heteromeric ATP-citrate lyase [77] and plants deficient in this activity display a complex, bonsai phenotype, with miniaturized organs, smaller cells, aberrant plastid morphology, reduced cuticular wax deposition, and hyperaccumulation of starch, anthocyanin, and stress-related mRNAs in vegetative tissue [77].

The cytosolic pool of malonyl-CoA is required for a variety of reactions including the elongation of very long-chain fatty acids (VLCFAs, $\geq$ C20), and the biosynthesis of flavonoids as the substrate of chalcone synthase and anthocyanin malonyltransferase. The plastid envelope is impermeable to malonyl-CoA. ACC1 null mutants are impaired in embryo morphogenesis and produce abnormal seeds which accumulate triacylglycerols lacking VLCFAs [78]. Recently, two ACC1 missense mutants, which were not embryo-lethal, were reported [79, 80]. One mutant line displays a freezing sensitive phenotype and the leaves are deficient in anthocyanin accumulation during cold acclimation. In oil seed rape leaves, decreasing the homomeric ACC activity by antisense RNA technology resulted in decreased 
accumulation of flavonoids under UV-B treatment which contrasts to their pattern of increase in the wild type [81]. The highest level of ACC1 mRNA was detected in flowers, where a large variety of flavonols are synthesized (Fig. 1) [82]. ACC activity has similarly been observed to be induced by strong light stress to parsley cell-suspension cultures [83], whilst homomeric ACC activity and transcripts were induced upon addition of yeast or fungal elicitors to alfalfa cell cultures [84]. Moreover, the expression of a set of flavonoid biosynthetic genes including cytosolic $A C C 1$ were induced upon UV-B stress in Arabidopsis [85]. Also in pea leaves, not the heteromeric ACC, but the homomeric form was induced by UV-B irradiation [86]. Taken together these results suggested cytosolic homomeric ACC is required and coordinately regulated with anthocyanin and isoflavone production under stress conditions.

\subsection{Formation of flavonoid scaffolds - the central flavonoid biosynthetic pathway}

Flavonoid scaffolds are formed from the building blocks of a phenylpropanoid primer and polyketide condensing units by a series of reactions including condensation, isomerization, oxidation and reduction, which are known to be involved in the central flavonoid biosynthetic pathway. The scaffold structures are further modified by tailoring reactions. In combination this suite of reactions defines the final individual compound structures.

Chalcone synthase (CHS) - Chalcone synthase (CHS) is the first committed enzyme in the biosynthesis of all flavonoids. This enzyme belongs to a family of type III polyketide synthase, which is one of the general polyketide synthases and forms a catalytically-active single polypeptide [87]. CHS catalyzes the so-called Claisen-ester condensation concomitant with $\mathrm{CO}_{2}$ liberation from malonyl-CoA and acyl-thioester (i.e., $p$-coumaroyl-CoA). Actually CHS catalyzes the formation of a triketide intermediate from $p$-coumaroyl-CoA and three molecules of malonyl-CoA; then spontaneous cyclization of the triketide intermediate results in the formation of naringenin chalcone [87]. There are three CHS-like genes forming a small gene family in A. thaliana. Among them, AtCHS (TT4, At5g13930) has been identified as the gene participating in the synthesis of flavonoids, and thus the mutant of this gene is designated as transparent testa ( $t$ t 4 since it lacks proanthocyanidin formation in the seed coat (Table 1) [88]. Two other genes, PKSA/LAP6 (At1g02050) and LAP5/PSKB (At4g34850), are known to encode hydroxyalkyl- $\alpha$-pyrone synthases which are essential for pollen development and the biosynthesis of sporopollenin, the constituent of exine in the outer pollen wall $[89,90]$. 
Chalcone isomerase (CHI) - Chalcone isomerase (CHI) catalyzes the stereospecific cyclization of naringenin chalcone to $(2 S)$-naringenin. In A. thaliana, there are five CHI-like genes. Among them, AtCHI (At3g55120) was identified as the TT5 gene [7, 10]. The expression of AtCHI-like1 (AtCHI-L1, At5g05270) was induced by the PAP1 transcription factor (AtMYB75, At1g56650) together with TT5, suggesting a functional similarity of these two genes [20]. A recent study indicated that non-catalytic CHI-like proteins localized in chloroplasts may function as fatty-acid-binding proteins necessary in proper production of fatty acids in developing seed embryos [91].

Flavanone 3-hydroxylase (F3H) - Flavanone 3-hydroxylase $(\mathrm{F} 3 \mathrm{H})$ is a 2-oxoglutarate-dependent dioxygenase catalyzing the oxygenation at 3-postion of flavanone ((2S)-naringenin) to form dihydroflavonol (dihydrokaempferol) with the concomitant production of $\mathrm{CO}_{2}$ and succinate from oxygen and 2-oxoglutarate as co-substrates. At3g51240 is known as the TT6 gene encoding F3H [92]. The activity of F3H in vivo can be partially compensated by two related 2-ODD enzymes in flavonoid biosynthesis, flavonol synthase (FLS) and leucoanthocyanidin dioxygenase /anthocyanidin synthase (LDOX/ANS) by the analysis of "leaky" phenotype of F3H mutant alleles [93]. This promiscuity of 2-ODDs involved in flavonoid biosynthesis has been also supported by the detailed mechanistic study of these reactions [51].

Flavonoid 3'-hydroxylase $\left(F^{\prime} H\right)$ - Flavonoid 3'-hydroxylase $\left(\mathrm{F}^{\prime} \mathrm{H}\right)$ is a cytochrome P450 monooxygenase committed in the hydroxylation of 3'-position of B-ring of flavonoid. This enzyme can accept either dihydrokaempferol or kaempferol as a substrate and convert them to dihydroquercetin and quercetin, respectively. At5g07990 which encodes CYP75B1 has been identified as the TT7 gene in A. thaliana [94].

Flavonol synthase (FLS) - Flavonol synthase (FLS) is the first committed enzyme for the biosynthesis of flavonols branching from the main trunk route to the branch for anthocyanin formation [95]. FLS catalyzing the double bond formation between $\mathrm{C}-2$ and $\mathrm{C}-3$ is one of three 2-ODD enzymes in the flavonoid biosynthesis pathway besides $\mathrm{F} 3 \mathrm{H}$ and LDOX/ANS. FLAVONOL SYNTHASE1 (AtFLS1, At5g08640), was first identified as the FLS gene encoding the catalytically active protein $[96,97]$. In the Arabidopsis genome, there are five additional genes with high sequence similarity to FLS. Although AtFLS1 predominantly influence flavonoid levels in Arabidopsis [98, 99], the mutant deficient AtFLS1 still accumulate significant amounts of flavonols. AtFLS3 (At5g63590) has been identified as a second active FLS [100]. The other FLS-like genes may have alternative noncatalytic functions, however these remain, as yet, unidentified. 
Dihydroflavonol reductase (DFR) - The first committed reaction leading to anthocyanin and proanthocyanidin is the reduction of the 4-keto group of dihydroflavonol to the corresponding leucoanthocyanidin. This reaction is catalyzed by dihydroflavonol reductase (DFR) competing the substrate, dihydroflavonol, with FLS. The gene At5g42800 corresponding to TT3, is known to encode functional DFR [101].

Leucoanthocyanidin dioxygenase /anthocyanidin synthase (LDOX/ANS) - The first colored compound in the anthocyanin biosynthetic pathway is anthocyanidin which exhibits a conjugated double bond system encompassing A, B and $\mathrm{C}$ rings. The enzyme LDOX/ANS catalyzes the formation of anthocyanidin from leucoanthocyanidin with 2-oxoglutarate and oxygen as co-substrates. In Arabidopsis, At4g22880 was assigned as TT18 it encodes the protein LDOX/ANS. The mutants, tt11, tt17 and tds 4 (tannin deficient seed 4), are also ascribed to mutation of this gene [102, 103]. The reaction mechanism of this enzyme has been investigated and was concluded to be a LDOX/ANS-catalyzed 2-oxoglutarate-dependent conversion of leucoanthocyanidin (flavan-3,4-cis-diol) to the pseudobase, 3-flaven-2,3-diol, most probably via 3-oxidation, 2,3-desaturation and isomerization, followed by stabilization by glucosylation at the C-3 position [104-106]. Three 2-oxoglutarate- and ferrous iron-dependent oxygenases involved in the flavonoid biosynthetic pathway, F3H, FLS and LDOX/ANS, are closely related by sequence and all catalyze oxidation of the C-ring. In fact, multifunctionality of FLS and LDOX/ANS has been indicated by several lines of evidence [107]. The apparent overlapping substrate and product selectivities of these three enzymes has been explained from the mechanistic view point [51]. The reactions catalyzed by these three enzymes commonly involve the oxidation of C-3 positions of C-ring of flavonoids by the oxygen atom activated by iron of the enzymes [51, 108].

Anthocyanidin reductase (ANR) - For the formation of proanthocyanidins (condensed tannins), anthocyanidin reductase (ANR) is the first committed enzyme in this pathway catalyzing the NADPH-dependent reduction of cyanidin to (-)-epicatechin (cis-flavan-3-ol). At1g61720 is designated as the BANYULS (BAN) gene encoding ANR [109-111]. The similar enzyme leucoanthocyanidin reductase (LAR) responsible for reduction of leucoanthocyanidin to catechin (trans-flavan-3-ol) has been reported in legume [33], grapevine [112] and poplar [113]. However, no gene with Arabidopsis homolog of $L A R$ has yet been characterized.

Polyphenol oxidase - Polyphenol oxidase (PPO), which is involved in the oxidative polymerization of (-)-epicatechin, has been characterized as a laccase-type enzyme designated TT10 (AtLAC15, At5g48100) [114, 115]. This enzyme is critical for the brown coloring of Arabidopsis seed coat as a function of the fact that it 
participates in a polymerization reaction. However, the precise mechanism for regiospecific oxidation of (-)-epicatechin molecule to $\mathrm{C} 4-\mathrm{C} 8$ oxidative linkage has not yet been clarified.

\subsection{Tailoring reactions of flavonoid scaffolds}

The huge chemical diversity of flavonoids is due to a high order modification by enzymes such as glycosyltransferases, methyltransferases and acyltransferases although structural variations within the flavonoid scaffold structures are relatively limited. These steps are known as tailoring reactions and they are responsible for final determination of physicochemical and biological properties of the flavonoid molecules which accumulated in cells.

Flavonoid glycosyltransferase (FGT) - Glycosylation is essential for stable accumulation of flavonoids in Arabidopsis and occurs at the C-3, C-5 and C-7 positions of flavonoid aglycones. Sugar moieties attached to flavonoid aglycones are also further glycosylated. Flavonoid glycosyltransferase (FGT) catalyzes the glycosylation of flavonoid skeletons. Some of the genes belonging to the family 1 glycosyltransferases (UGTs) which utilize UDP-sugars as sugar donors for glycosylation, have been characterized as FGTs in Arabidopsis. In the Arabidopsis genome there are, depending on the report taken, between 107 and 120 genes encoding family 1 glycosyltransferases $[52,116,117]$. The detailed analysis of flavonoid structures suggest that at least ten FGTs are involved in flavonoid glycosylation. So far, seven genes encoding FGTs were identified (Table 1). Generally, 3-O-glycosylation is known to have occurred prior to glycosylation of other position (Figure 3). As 3-O-glycosyltransferases which specifically glycosylate at C-3 position of flavonol aglycone but not anthocyanidin, two enzymes, flavonol 3-O-rhamnosyltransferase (UGT78D1, Atlg30530) [118] and flavonol 3-O-arabinosyltransferase (UGT78D3, At5g17030) [25], were identified in Arabidopsis. However, flavonoid 3-O-glucosyltransferase (UGT78D2, At5g17050) can transfer glucose to the C-3 position of both flavonol and anthocyanin aglycones [20]. A mutant lacking functional flavonoid 3-O-glucosyltransferase was characterized as exhibiting drastically decreased anthocyanin contents, yet still accumulated a small amount of the anthocyanin A11. These data indicate that 3-O-glucosylation by UGT78D2 plays a major role in the stable accumulation of anthocyanins and it furthermore seems likely that Arabidopsis has additional gene(s) encoding anthocyanin 3-O-glucosyltransferase(s) [20]. Anthocyanin 5-O-glucosyltransferase (UGT75C1, At4g14090), flavonol 7-O-glucosyltransferase (UGT73C6, At2g36790) and flavonol 
7-O-rhamnosyltransferase (UGT89C1, At1g06000) have also been identified [20, 118, 119]. The mutation of anthocyanin 5-O-glucosyltransferase resulted in the accumulation of anthocyanins lacking a glucose moiety at the C-5 position [20]. In the ugt73c6 knockout mutant, flavonol 7-O-glucosides were missing. On the other hand, ugt $89 \mathrm{cl}$ mutants accumulated unknown flavonols. UGT79B1 (At5g54060) is an anthocyanin 3-O-glucoside: 2"-O-xylosyltransferase [120]. Total anthocyanin is drastically decreased in ugt79b1 knockout mutants, suggesting that additional 2"-O-xylosylation of 3-O-glucoside of cyanidin is required for stable anthocyanin accumulation in Arabidopsis (Figure 4). Recently, it was additionally found that glycoside hydrolase (GH)-type FGTs transfer the sugar moiety from an acyl-glucose to anthocyanins in Dianthus caryophyllus (carnation) and Delphinium grandiflorum (delphinium) [121, 122]. The Arabidopsis ortholog, AtBGLU10 (At4g27830) is presumably involved in glucosylation of the coumaroyl moiety on anthocyanin A11 [123]. This GH-type FGT reaction occurs inside vacuoles resulting in the storage form of anthocyanins.

Phylogenetic analyses of FGTs indicated that FGTs form distinct clusters on the basis of their regioselectivity for glycosylation [124]. When considered from an evolutionary perspective, Arabidopsis UGTs can be divided into 12 groups based on phylogenetic analysis [116]. More extensive analysis on UGTs from six plant species (Arabidopsis thaliana, A. lyrata, Populus trichocarpa, Oryza sativa, Selaginella moellendorffii, Physcomitrella patens) indicated that these UGTs can be classified into 24 orthologous groups [52]. An orthologous group is defined as a group containing genes that have diverged in each species from those of their common ancestor. In both instances, flavonoid 3-O-glycosyltransferases form a distinct group, suggesting that the function of flavonoid 3-O-glycosyltransferases was established before the divergence from $P$. patens and thus flavonoid 3-O-glycosyltransferases acquired substrate specificity for sugar donor following plant specification. The UGT families can be classified into 24 orthologous groups, which are defined as groups containing genes that had diverged in each species from those of their common ancestor [52]. FGTs except for flavonoid 3-O-glycosyltransferases belong to groups including multiple UGT subfamilies. For example, anthocyanin 5-O-glucosyltransferase (UGT75C1) belongs to a group consisting of UGT74, UGT75 and UGT84 subfamilies. UGT74s and UGT84s are involved in glucosinolate biosynthesis and phenylpropanoid modification/ auxin deactivation, respectively. During the branching processes to UGT74s, UGT75s and UGT84s from a common ancestor, UGT75C1 may have acquired the substrate specificity for anthocyanin. Thus, FGTs except for flavonoid 3-O-glycosyltransferases may have gained the substrate specificity for flavonoids independently. 
Flavonoid methyltransferase (FMT) - In Arabidopsis, there are 24 SABATH-type methyltransferase genes [125]. AtOMT1 (At5g54160) has been characterized as being involved in the methylation of flavonols forming isorhamnetin a fact supported by the evidence from in vivo [24] and in vitro experimental evidence [126]. AtOMT1 also participates in the production of lignins and sinapoyl esters [127].

Flavonoid acyltransferase (FAT) - Acyltransferases can also be classified within the BAHD and serine carboxypeptidase-like (SCPL) families. The BAHD family was named after the first four biochemically characterized enzymes in this family (BEAT, AHCT, HCBT, and DAT) and all use acyl coenzyme A thioesters as acyl donors [128]. By contrast, the SCPL family use acyl-activated sugars such as $1-O$ - $\beta$-glucose esters [129]. BAHD-type acyltransferases are predicted to be localized in cytosol (or cytoplasmic surface of the ER) whereas SCPL-type acyltransferases are vacuolar enzymes [124].

The Arabidopsis genome contains 64 and 53 genes encoding BAHD- and SCPL-type acyltransferases, respectively [117] and of these two BAHD-type and one SCPL-type acyltransferases are involved in flavonoid acylation. Anthocyanin 5-O-glucoside malonyltransferase (At3g29590) and anthocyanin 3-O-glucoside coumaroyltransfreases (Atlg03940 and At1g03495) have been identified as BAHD type acyltransferases and these acylations contribute to the stability of anthocyanins at neutral $\mathrm{pH}$ [130]. The gene At2g23000 was identified as an SCPL-type acyltransferase specifically functioning as an anthocyanin 3-O-glucoside-2"- $O$-xyloside: sinapoyltransferase [131].

\section{The anthocyanin modification pathway in Arabidopsis}

The pathway from cyanidin to the most highly modified anthocyanin A11 requires seven modification steps by four glycosyltransferases and three acyltransferases (Figure 5). Enzymatic characterization has been carried out for all modifying enzymes with the exception of cyanidin 3-(6"-p-coumaroylglucoside): glucosyltransferase, facilitating a fairly complete overview of the major routes of anthocyanin modification.

Flavonoid 3-O-glucosyltransferase is the first committed modification enzyme $[20,132]$. Anthocyanin 3-O-glucoside: 2"- $O$-xylosyltransferase recognizes cyanidin 3-O-glucoside, but not cyanidin 3,5-O-diglucoside as a substrate. Anthocyanin 3-O-glucoside: 6"-O-coumaroyltransferase prefers cyanidin 3-O-glucoside, but shows very minor activity for cyanidin 3,5-O-diglucoside. These data strongly suggest that 
$2 "-O$-xylosylation and 6"-coumaroylation occur prior to 5-O-glucosylation. This is also supported by the fact that the recombinant UGT75C1 failed to 5-O-glucosylate cyanidin 3-O-glucoside (Y. Nishiyama, T. Tohge, M. Yamazaki and K. Saito, unpublished data), similarly as the homologous Petunia hybrid enzyme was unable [133]. These data suggest that UGT75C1 prefers 2"-O-xylosylated anthocyanins as substrates. In sharp contrast, the properties of anthocyanin 5-O-glucosyltransferases from Perilla frutescens and Verbena hybrida revealed that both exhibited a broad substrate specificity accepting cyanidin 3-O-glucoside besides bis-glycosylated anthocyanins [134]. Anthocyanin malonyltransferase and sinapoyltransferase are a BAHD-type and an SCPL-type acyltransferase, respectively. The sub-cellular localization of these acyltransferases suggests that cytosolic malonyltransferase acts prior to the vacuolar sinapoyltransferase. Thus, cyanidin is thought to be modified following the order 3-O-glucosylation, xylosylation or coumaroylation, 5-O-glucosylation and finally malonylation.

Recently, in addition to FGTs and FATs, it was reported that several enzymes affect flavonoid modification pathway although they do not interact directly with flavonoid molecules. For example sinapate glucosyltransferase, AtSGT1 (bright trichomes 1, AtBRT1, At3g21560, UGT84A2), catalyzes the conversion of sinapate to 1-O-sinapoyl glucose, an acyl donor for acyltransferases [135]. In ugt84a2 knockout mutants, a major sinapoylated anthocyanin A11 is drastically decreased and instead, a non-sinapoylated anthocyanin A5 (cyanidin 3-O-[2"-O-(xylosyl)-6"-O-( $p$-coumaroyl) glucoside] 5-O-malonylglucoside, Figure 5A) is detected as the major anthocyanin [120]. This suggests that UGT84A2 is a major supplier of the sinapoyl moiety for anthocyanins and that limited supply of 1-O-sinapoyl glucose as a consequence of the mutation in ugt84a2 suppresses production of sinapoylated anthocyanins. Intriguingly, in the sng1-5 mutant (deletion in genes encoding sinapoylglucose:malate sinapoyltransferase 1, AtSNG1, At2g22990 and anthocyanin 3-O-[2"-O-(xylosyl)-glucoside sinapoyltransferase, SAT, At2g23000), anthocyanin A8 (cyanidin 3-O-[2"-O-(xylosyl) 6"-O-(p-O-(glucosyl) $\quad p$-coumaroyl) glucoside] $5-O-[6 " '-O$-(malonyl) glucoside $]$, Figure $5 \mathrm{~A}$ ) accumulated as the major anthocyanin [136]. The difference in the major anthocyanin species in ugt84a2 and sng1-5 mutants suggests that: (1) sinapoyltransferase may have a higher affinity to A5 than anthocyanin 3-O-6"-O-coumaroylglucoside: glucosyltransferase and thus inhibits further glucosylation of A5 as a consequence of tight binding of A5 in ugt84a2 mutants or (2) anthocyanin 3-O-6"-O-coumaroylglucoside: glucosyltransferase utilizes 1-O-sinapoyl glucose as sugar donor and the glucosyltransferase activity may be also suppressed by the limited supply of 1-O-sinapoyl glucose in ugt84a2 mutants. 


\section{Flavonol modification pathway in Arabidopsis}

Based on the flavonoid structures identified to date it would appear that the flavonol modification pathway needs three steps at the most (Figure 3). 3-O-Glycosylation occurs first. In the case of flavonols, the $\mathrm{C}-3$ position is glycosylated with glucose, rhamnose or arabinose. Therefore, mutation of one of 3-O-glycosyltransferase genes would result in the increase of flavonols glycosylated by other flavonol 3-O-glycosyltransferases. The order of flavonol 7-O-glycosyltransferase and glycosyltransferase which glycosylate the sugar moiety attached to flavonol aglycones (GGTs) is unknown. However, anthocyanin GGTs prefer anthocyanin 3-O-glucoside over anthocyanidin 3,5-O-diglucosides [120, 137, 138]. It suggests that flavonol GGTs may also prefer flavonol 3-O-glucoside over flavonol 3-O-glucoside-7-O-glycosides.

UDP-rhamnose synthase 1 (RHM1, AtROL1, At1g78570) was identified to be an enzyme which affects flavonol modification pathway even though it does not interact directly with flavonol molecules [25]. RHM1 was isolated as a gene whose expression manner is highly correlated with those of flavonoid biosynthetic genes. In rhm 1 knockout mutants, flavonol 3-O-rhamnoside derivatives are significantly decreased and flavonol 3-O-glucooside derivatives are increased. Arabidopsis has three UDP-rhamnose synthase genes (RHM1; RHM2, AtMUM4, At1g53500; RHM3, At3g14790) [139-141]. Comparison of the flavonol profiles in RHM knockout mutants indicated that RHM1 plays a major role in supply of the UDP-rhamnose precursors for the flavonol modification pathway. Interestingly the manner of flavonol 3-O-glycosylation is more affected by the limitation of UDP-rhamnose supply than 7-O-glycosylation is.

\section{Subcellular translocation of flavonoid in plants}

In addition to the structural enzymes discussed above transport proteins are also essential for flavonoid biosynthesis. Functional characterization of the screened mutant series named either $t t$ or $t d s$ has led to a better understanding of flavonoid transport proteins and their transport mechanism [88, 103, 142]. Proanthocyanidin is thought to be stored in the vacuoles of seed coat endothelial cells following oligomerization and polymerization of PA intermediates which are transported from the cytosolic face of the endoplasmic reticulum (ER) $[143,144]$. Characterization of the 
tt12 mutant encoding a membrane protein of the multi-drug and toxic efflux (MATE) transporter family revealed that the TT12 protein (At3g59030) is related to vacuolar transport of proanthocyanidin in Arabidopsis seed coats [145]. However, whilst TT12 protein when expressed in yeast could transport cyanidin-3-O-glucoside it could not transport cathechin-3-O-glucoside, flavonol glycosides or proanthocyanidin dimers [146]. Furthermore, although there is evidence that catechin-3-O-glucoside inhibits TT12-mediated transport of cyanidin-3-O-glucoside, flavan-3-ol and flavonol glycosides had no effect on anthocyanin transport. This fact suggests that transport catalyzed by the TT12 protein is confined to flavan-3-ol-glycosides [146]. The flower flavonoid transporter (FFT, AtDTX35), a member of the MATE family, was isolated by the identification of the mutation resulting in floral and growth phenotypes and reduction of floral kaempferol di-glucoside. The expression of this gene is highly localized to epidermal guard cells in flower tissues [147].

Over 15 years ago a glutathione $S$-transferase-like (GST) was identified as an essential protein for anthocyanin and proanthocyanidin transport to the vacuole. This protein, also known as Bronze2, was isolated from an anthocyanin deficient mutant (bz2) in maize [148]. Interestingly, TT19 (TT14, GSTF12, At5g17220) also encodes an Arabidopsis GST-like protein [143]. In Arabidopsis, there are three GST-like genes, which are up-regulated with $P A P 1$ overexpression and their gene products exhibited a weak GST activity [149]. However, TT19 is almost exclusively involved in anthocyanin accumulation. To date several flavonoids related GST-like genes have been found in diverse plant species. Plant flavonoid related GST-like genes being found across the different types of GST family which are sub-divided on the basis of sequence identify, for example, maize Bz2 [148], soybean GmGST26A [150] and parsley PcGST1 [151] belong to the Tau type GSTs whereas petunia $A N 9$ [150, 152], cyclamen [153] and Arabidopsis TT19 [143] belong to the Phi type GSTs [154]. Furthermore, petunia AN9 could complement the anthocyanin accumulation of the Arabidopsis $t t 19$ mutant but not its deficiency in proanthocyanidin production. Despite the relatively common identification of changes in flavonoids in GST-like mutants the function of GST-like in the transport processes is still very much ambiguous [152, 155] since whilst the GST-likes are themselves directly involved as components of the flavonoid transport system despite their name the glutathione molecule itself may not be required for flavonoid transport. This fact notwithstanding it is clear that GST-like function in the cytosol in order to maintain regular accumulation of proanthocyanidin precursors such as epicatechin and epicatechin-glycosides in the vacuole $[144,156]$. Recent study with a TT19-GFP fusion protein indicated that TT19 is localized not only in the cytoplasm and 
nuclei, but also on the tonoplast [157]. Flavonol specific GST-like proteins have not yet been identified, however one candidate GST-like, At1g10370, has been discussed in the context of belonging to the co-expression network of flavonol biosynthetic genes [25]. Further genetic and biochemical work is however required in order to confirm the hypothesized function of this gene.

Arabidopsis AHA10 (TT13, Atlg17260), a putative P-type $\mathrm{H}^{+}$-ATPase been isolated from the aha10 mutant which shows vacuolar morphological defects and reduction of proanthocyanidin accumulation levels in seeds [10, 158]. This enzyme is considered to act in tandem with the activity of the MATE transporter TT12 to maintain a $\mathrm{H}^{+}$/flavonoid-antiport function in the Arabidopsis. Interesting although the $t t 12$ knockout mutant accumulates epicatechin-glycosides in seeds a considerably higher level of epicatechin was observed in ahal0 mutant seeds [32]. AHA10 is thought to be localized on the cytosolic face of the ER or on vesicles involved in PA transport from the cytosol to the vacuole and it is hence hypothesized that vesicle trafficking-mediated PA transport may require AHA10 to supply energy [144].

\section{Future prospects}

The majority of chemical diversity of flavonoid molecules in Arabidopsis has been characterized by the efforts of the decade. The genes involved in the synthesis of these diverse molecules have been also intensively identified by use of cutting-edge functional genomics approaches [159], although several missing genes/enzymes need to be identified and subsequently characterized further. However, the biological significance of this enormous diversification of flavonoids during developmental changes and respective ecotype specificity should be addressed by further investigation. The functionality of diversity could be related to the adaptation of Arabidopsis plants to environments during the evolution. These findings could be the basis for further engineering of flavonoids [160] and optimization of the metabolic pathway [161]. The study of flavonoid metabolism in Arabidopsis should additionally be extended to non-model medicinal or exotic plant species producing flavonoids or even other pharmacologically-active compounds [162-164]. Biotechnological application by transgenic technology for the promotion of human health by engineering of flavonoid is based on an in-depth understanding of fundamental mechanism of the biosynthetic mechanisms is also a promising area for future research $[165,166]$. Relatively inexpensive high-throughput sequencing technology in combination with emerging metabolomics technologies $[167,168]$ will likely facilitate the mining of yet more genes 
involved in this specialized metabolic pathway of plants [169].

Acknowledgements - The authors' research was supported, in part, by a Grant-in-Aid for Scientific Research on Innovative Areas from The Ministry of Education, Culture, Sports, Science and Technology (MEXT), Japan, Strategic International Collaborative Research Program (SICORP) and Strategic International Research Cooperative Program (SICP) of Japan Science and Technology Agency (JST). 
Table 1. Genes for the flavonoid biosynthesis

\begin{tabular}{|c|c|c|c|c|}
\hline Protein function & $\begin{array}{l}\text { Gene } \\
\text { designation }\end{array}$ & $\begin{array}{l}\text { transparent testa } \\
\text { mutant }\end{array}$ & Locus & Ref. \\
\hline \multirow[t]{2}{*}{ phenylalanine ammonia-lyase } & PAL1 & & At2g37040 & {$[58]$} \\
\hline & PAL2 & & At3g53260 & $\lceil 59\rceil$ \\
\hline cinnamic acid 4-hydroxylase & $\mathrm{C} 4 \mathrm{H}$ & & At2g 30490 & $\lceil 64\rceil$ \\
\hline 4-coumaric acid:CoA ligase & $4 \mathrm{CL} 3$ & & At1g65060 & $\lceil 67\rceil$ \\
\hline acetyl-CoA carboxylase & $\mathrm{ACC} 1$ & & At1g36160 & $\lceil 73,74\rceil$ \\
\hline chalcone synthase & CHS & $t t 4$ & At5g 13930 & $\lceil 88\rceil$ \\
\hline \multirow[t]{2}{*}{ chalcone isomerase } & $\mathrm{CHI}$ & $t t 5$ & At3g55120 & $\lceil 88\rceil$ \\
\hline & CHI-L1 & & At5g05270 & $\lceil 20\rceil$ \\
\hline flavanone 3-hydroxylase & $\mathrm{F} 3 \mathrm{H}$ & $t t 6$ & At3g51240 & $\lceil 92\rceil$ \\
\hline flavonoid 3'-hydroxylase & F3'H & $t t 7$ & At5g07990 & $\lceil 94\rceil$ \\
\hline \multirow[t]{2}{*}{ flavonol synthase } & FLS1 & & At5g08640 & $\lceil 95\rceil$ \\
\hline & FLS3 & & At5g63590 & {$[98,100\rceil$} \\
\hline dihydroflavonol reductase & DFR & $t t 3$ & At5g 42800 & $\lceil 101\rceil$ \\
\hline leucoanthocyanidin dioxygenase/anthocyanidin & LDOX/ANS & $t t 18, t t 11, t t 17$ & At4g 22880 & [109] \\
\hline anthocaynin reductase & ANR & ban (banyuls) & At1g61720 & $\lceil 111\rceil$ \\
\hline laccase & LAC15 & $t t 10$ & At5g48100 & $\lceil 114\rceil$ \\
\hline glutathione $S$-transferase & GSTF12 & $t t 19, t t 14$ & At5g 17220 & $\lceil 10,143\rceil$ \\
\hline multi-drug and toxic efflux (MATE) transporter & TT12 & $t t 12$ & At3g59030 & $\lceil 145\rceil$ \\
\hline P-type $\mathrm{H}^{+}$-ATPase & AHA10 & tt13, aha10 & Atlg17260 & $\lceil 10,158\rceil$ \\
\hline flavonoid 3-O-glucosyltransferase & F3GlcT & & At5g 17050 & $\lceil 20,132\rceil$ \\
\hline flavonol 3-O-rhamnosyltransferase & F3RhaT & & At1g30530 & $\lceil 118\rceil$ \\
\hline flavonol 3-O-arabinosyltransferase & F3AraT & & At5g 17030 & $\lceil 25\rceil$ \\
\hline flavonol 7-O-glucosyltransferase & F7GlcT & & At2g 36790 & $\lceil 118\rceil$ \\
\hline flavonol 7-O-rhamnosyltransferase & F7RhaT & & At1g06000 & $\lceil 119\rceil$ \\
\hline anthocyanin 5-O-glucosyltransferase & A5GlcT & & At4g 14090 & $\lceil 20\rceil$ \\
\hline anthocyanin 3-O-glucoside: 2 "-O-xylosyltransferase & A3G2"XylT & & At5g54060 & $\lceil 20,120\rceil$ \\
\hline anthocyanin 3-O-6"-O-coumaroylglucoside: & BGLU10 & & At4g27830 & {$[123]$} \\
\hline \multicolumn{5}{|l|}{ glucosyltransferase } \\
\hline methyltransferase & OMT1 & & At5g54160 & $\lceil 24,126\rceil$ \\
\hline anthocyanin malonyltransferase (BAHD) & A5GlcMalT & & At3g29590 & $\lceil 125\rceil$ \\
\hline \multirow[t]{2}{*}{ anthocyanin coumaroyltransferase (BAHD) } & A3GlcCouT & & At1g03495 & $\lceil 20,130\rceil$ \\
\hline & A3GlcCouT & & At1g03940 & $\lceil 20,130\rceil$ \\
\hline anthocyanin sinapoyltransferase (SCPL) & A3Glc2"XylSinT & & At2g23000 & {$[131]$} \\
\hline
\end{tabular}




\section{Legends to figures}

Figure 1. Flavonoid diversity in Arabidopsis.

(A) Structures of anthocyanins, flavonols and proanthocyanidins detected in A. thaliana.

(B) Accumulation sites of flavonoids in Arabidopsis tissues. Grey color indicates the sites where the flavonoid molecules were detected. Key: C, cyanidin; K, kaempferol; Q, quercetin; I, isorhamnetin; E, epicatechin; mal, malonyl; $p$-cou, $p$-coumaroyl; sina, sinapoyl; glc, glucose; rha, rhamnose; ara, arabinose; hex, hexose; deoxyhex, deoxyhexose; pent, pentose. Flavonoid derivatives were found in Arabidopsis leaves [18, $20,23,25,28,30,119]$, root $[20,24,25,28,29]$, flowers $[15,25,28]$ and seeds $[10,21$, $22,28,32]$ by LC-MS or LC-UV/VIS-MS studies, and identified or characterized by 1D- and 2D-NMR, IR, UV and MS studies [17, 19, 25, 26].

Figure 2. General biosynthetic pathway of phenylpropanoid and flavonoid skeletons.

PAL, phenylalanine ammonia-lyase; $\mathrm{C} 4 \mathrm{H}$, cinnamic acid 4-hydroxylase; 4CL, 4-coumaric acid: CoA ligase; ACC, acetyl-CoA carboxylase; CHS, chalcone synthase; $\mathrm{CHI}$, chalcone isomerase; $\mathrm{F} 3 \mathrm{H}$, flavanone 3-hydroxylase; $\mathrm{F} 3{ }^{\prime} \mathrm{H}$, flavonoid 3'-hydroxylase; FLS, flavonol synthase; OMT1, O-methyltransferase 1; DFR, dihydroflavonol 4-reductase; ANS, anthocyanidin synthase; ANR, anthocyanidin reductase.

\section{Figure 3. Proposed flavonol modification pathway in Arabidopsis}

The compounds detected in Arabidopsis are shown. White arrows indicate proposed but unidentified pathways. OMT1, $O$-methyltransferase 1; F3AraT, flavonol 3-O-arabinosyltransferase; F3GlcT, flavonoid 3-O-glucosyltransferase; F3RhaT, flavonol 3-O-rhamnosyltransferase; F7GlcT, flavonol 7-O-glucosyltransferase; F7RhaT, flavonol 7-O-rhamnosyltransferase.

\section{Figure 4. Ds transposon insertion mutants of UGT79B1}

(A) Phenotype of the wild-type (F-Nossen), Ds53, Ds54 and Arabidopsis transposon tagged lines, ugt79b1-1 (Ds53-4592-1) and ugt79b1-2 (Ds-54-1263-1). Plants were grown on one-half-strength Ms-agar medium containing 5\% sucrose in a growth chamber at $22^{\circ} \mathrm{C}$ with $16 \mathrm{~h} / 8 \mathrm{~h}$ light and dark cycles for 7 days.

(B) HPLC/PDA/MS analyses of the ugt79b1 mutant lines.* a compound which has the 
$m / z \quad$ value corresponding to cyanidin 3-O-(6"-O-p-coumaroylglucoside)-5-O-(6"-O-malonylglucoside); ** a compound which has the $m / z$ value corresponding to cyanidin 3-O-6"-O-p-coumaroylglucoside.

\section{Figure 5. Proposed anthocyanin modification pathway}

The metabolic grid for the biosynthesis of A11. Black and pale grey arrows indicate potential glucosylation (Glc), xylosylation ( $\mathrm{Xyl}$ ), coumaroylation ( $\mathrm{Cou}$ ), sinapoylation (Sin), and malonylation (Mal). Dark grey arrows on the metabolic grid indicate putative major anthocyanin modification route. $\mathrm{C} 3 \mathrm{G}$, cyanidin 3-O-glucoside; $\mathrm{C} 3 \mathrm{G} 5 \mathrm{G}$, cyanidin 3,5-O-diglucoside; $\mathrm{C} 3 \mathrm{G} 2 " \mathrm{X}$, cyanidin 3-O-xylosyl $(1 \rightarrow 2)$ glucoside. 


\section{References cited:}

[1] E. Haslam, Shikimic Acid: Metabolism and Metabolites, Shikimic Acid, John Wiley \& Sons, Chichester, UK (1993) 156-254.

[2] B. Halliwell, J. Rafter, A. Jenner, Health promotion by flavonoids, tocopherols, tocotrienols, and other phenols: direct or indirect effects? Antioxidant or not?, The American Journal of Clinical Nutrition, 81 (2005) 268S-276S.

[3] L. Wang, I.-M. Lee, S.M. Zhang, J.B. Blumberg, J.E. Buring, H.D. Sesso, Dietary intake of selected flavonols, flavones, and flavonoid-rich foods and risk of cancer in middle-aged and older women, The American Journal of Clinical Nutrition, 89 (2009) 905-912.

[4] A.T. Jan, M.R. Kamli, I. Murtaza, J.B. Singh, A. Ali, Q.M.R. Haq, Dietary Flavonoid Quercetin and Associated Health Benefits-An Overview, Food Reviews International, 26 (2010) 302-317.

[5] R.J. Bino, R.D. Hall, O. Fiehn, J. Kopka, K. Saito, J. Draper, B.J. Nikolau, P. Mendes, U. Roessner-Tunali, M.H. Beale, R.N. Trethewey, B.M. Lange, E.S. Wurtele, L.W. Sumner, Potential of metabolomics as a functional genomics tool, Trends in Plant Science, 9 (2004) 418-425.

[6] K. Saito, F. Matsuda, Metabolomics for functional genomics, systems biology, and biotechnology, Annual Review of Plant Biology, 61 (2010) 463-489.

[7] B. Winkel-Shirley, Flavonoid Biosynthesis. A Colorful Model for Genetics, Biochemistry, Cell Biology, and Biotechnology, Plant Physiology, 126 (2001) 485-493.

[8] E. Grotewold, The genetics and biochemistry of floral pigments, Annual Review of Plant Biology, 57 (2006) 761-780.

[9] M.K. Davies, K.E. Schwinn, Molecular biology and biotechnology of flavonoid biosynthesis, in: O.M. Andersen, K.R. Markham (Eds.) FLAVONOIDS Chemistry, Biochemistry abd Applications, CRC Press, Taylor \& Francis, Boca Raton, 2006, pp. 143-218.

[10] L. Lepiniec, I. Debeaujon, J.-M. Routaboul, A. Baudry, L. Pourcel, N. Nesi, M. Caboche, Genetics and biochemistry of seed flavonoids, Annual Review of Plant Biology, 57 (2006) 405-430.

[11] M.A. Farag, D.V. Huhman, Z. Lei, L.W. Sumner, Metabolic profiling and systematic identification of flavonoids and isoflavonoids in roots and cell suspension cultures of Medicago truncatula using HPLC-UV-ESI-MS and GC-MS, Phytochemistry, 68 (2007) 342-354.

[12] H. Suzuki, R. Sasaki, Y. Ogata, Y. Nakamura, N. Sakurai, M. Kitajima, H. Takayama, S. 
Kanaya, K. Aoki, D. Shibata, K. Saito, Metabolic profiling of flavonoids in Lotus japonicus using liquid chromatography Fourier transform ion cyclotron resonance mass spectrometry, Phytochemistry, 69 (2008) 99-111.

[13] F.S. Hosseinian, W. Li, T. Beta, Measurement of anthocyanins and other phytochemicals in purple wheat, Food Chemistry, 109 (2008) 916-924.

[14] H. Horai, M. Arita, S. Kanaya, Y. Nihei, T. Ikeda, K. Suwa, Y. Ojima, K. Tanaka, S. Tanaka, K. Aoshima, Y. Oda, Y. Kakazu, M. Kusano, T. Tohge, F. Matsuda, Y. Sawada, M.Y. Hirai, H. Nakanishi, K. Ikeda, N. Akimoto, T. Maoka, H. Takahashi, T. Ara, N. Sakurai, H. Suzuki, D. Shibata, S. Neumann, T. Iida, K. Tanaka, K. Funatsu, F. Matsuura, T. Soga, R. Taguchi, K. Saito, T. Nishioka, MassBank: a public repository for sharing mass spectral data for life sciences, Journal of Mass Spectrometry, 45 (2010) 703-714.

[15] F. Matsuda, K. Yonekura-Sakakibara, R. Niida, T. Kuromori, K. Shinozaki, K. Saito, MS/MS spectral tag-based annotation of non-targeted profile of plant secondary metabolites, Plant Journal, 57 (2009) 555-577.

[16] Y. Sawada, R. Nakabayashi, Y. Yamada, M. Suzuki, M. Sato, A. Sakata, K. Akiyama, T. Sakurai, F. Matsuda, T. Aoki, M.Y. Hirai, K. Saito, RIKEN tandem mass spectral database (ReSpect) for phytochemicals: A plant-specific MS/MS-based data resource and database, Phytochemistry, 82 (2012) 38-45.

[17] T.L. Graham, Flavonoid and flavonol glycoside metabolism in Arabidopsis, Plant Physiology and Biochemistry, 36 (1998) 135-144.

[18] M. Veit, G.F. Pauli, Major flavonoids from Arabidopsis thaliana leaves, Journal of Natural Products, 62 (1999) 1301-1303.

[19] S.J. Bloor, S. Abrahams, The structure of the major anthocyanin in Arabidopsis thaliana, Phytochemistry, 59 (2002) 343-346.

[20] T. Tohge, Y. Nishiyama, M.Y. Hirai, M. Yano, J. Nakajima, M. Awazuhara, E. Inoue, H. Takahashi, D.B. Goodenowe, M. Kitayama, M. Noji, M. Yamazaki, K. Saito, Functional genomics by integrated analysis of metabolome and transcriptome of Arabidopsis plants over-expressing an MYB transcription factor, Plant Journal, 42 (2005) 218-235.

[21] L. Kerhoas, D. Aouak, A. Cingoz, J.-M. Routaboul, L. Lepiniec, J. Einhorn, N. Birlirakis, Structural characterization of the major flavonoid glycosides from Arabidopsis thaliana seeds, Journal of Agricultural and Food Chemistry, 54 (2006) 6603-6612.

[22] J.M. Routaboul, L. Kerhoas, I. Debeaujon, L. Pourcel, M. Caboche, J. Einhorn, L. Lepiniec, Flavonoid diversity and biosynthesis in seed of Arabidopsis thaliana, Planta, 224 (2006) 96-107.

[23] M. Stobiecki, A. Skirycz, L. Kerhoas, P. Kachlicki, D. Muth, J. Einhorn, B. 
Mueller-Roeber, Profiling of phenolic glycosidic conjugates in leaves of Arabidopsis thaliana using LC/MS, Metabolomics, 2 (2006) 197-219.

[24] T. Tohge, K. Yonekura-Sakakibara, R. Niida, A. Watanabe-Takahashi, K. Saito, Phytochemical genomics in Arabidopsis thaliana: A case study for functional identification of flavonoid biosynthesis genes, Pure and Applied Chemistry, 79 (2007) 811-823.

[25] K. Yonekura-Sakakibara, T. Tohge, F. Matsuda, R. Nakabayashi, H. Takayama, R. Niida, A. Watanabe-Takahashi, E. Inoue, K. Saito, Comprehensive flavonol profiling and transcriptome coexpression analysis leading to decoding gene-metabolite correlations in Arabidopsis, Plant Cell, 20 (2008) 2160-2176.

[26] R. Nakabayashi, M. Kusano, M. Kobayashi, T. Tohge, K. Yonekura-Sakakibara, N. Kogure, M. Yamazaki, M. Kitajima, K. Saito, H. Takayama, Metabolomics-oriented isolation and structure elucidation of 37 compounds including two anthocyanins from Arabidopsis thaliana, Phytochemistry, 70 (2009) 1017-1029.

[27] T. Tohge, A.R. Fernie, Web-based resources for mass-spectrometry-based metabolomics: A user's guide, Phytochemistry, 70 (2009) 450-456.

[28] F. Matsuda, M.Y. Hirai, E. Sasaki, K. Akiyama, K. Yonekura-Sakakibara, N.J. Provart, T. Sakurai, Y. Shimada, K. Saito, AtMetExpress Development: A Phytochemical Atlas of Arabidopsis Development, Plant Physiology, 152 (2010) 566-578.

[29] P. Vallabhaneni, W.K. Ray, B.S.J. Winkel, R.F. Helm, Characterization of flavonol glycosides in individual Arabidopsis root tips by flow injection electrospray mass spectrometry, Phytochemistry, 73 (2012) 114-118.

[30] D.D. Rowan, M. Cao, K. Lin-Wang, J.M. Cooney, D.J. Jensen, P.T. Austin, M.B. Hunt, C. Norling, R.P. Hellens, R.J. Schaffer, A.C. Allan, Environmental regulation of leaf colour in red 35S:PAP1 Arabidopsis thaliana, New Phytologist, 182 (2009) 102-115.

[31] R. Stracke, O. Jahns, M. Keck, T. Tohge, K. Niehaus, A.R. Fernie, B. Weisshaar, Analysis of PRODUCTION OF FLAVONOL GLYCOSIDES-dependent flavonol glycoside accumulation in Arabidopsis thaliana plants reveals MYB11-, MYB12-and MYB111-independent flavonol glycoside accumulation, New Phytologist, 188 (2010) 985-1000.

[32] S. Kitamura, F. Matsuda, T. Tohge, K. Yonekura-Sakakibara, M. Yamazaki, K. Saito, I. Narumi, Metabolic profiling and cytological analysis of proanthocyanidins in immature seeds of Arabidopsis thaliana flavonoid accumulation mutants, Plant Journal, 62 (2010) 549-559.

[33] G.J. Tanner, K.T. Francki, S. Abrahams, J.M. Watson, P.J. Larkin, A.R. Ashton, Proanthocyanidin biosynthesis in plants - Purification of legume leucoanthocyanidin 
reductase and molecular cloning of its cDNA, Journal of Biological Chemistry, 278 (2003) 31647-31656.

[34] D.Y. Xie, R.A. Dixon, Proanthocyanidin biosynthesis - still more questions than answers?, Phytochemistry, 66 (2005) 2127-2144.

[35] Y. Pang, G.J. Peel, E. Wright, Z. Wang, R.A. Dixon, Early steps in proanthocyanidin biosynthesis in the model legume Medicago truncatula, Plant Physiology, 145 (2007) 601-615.

[36] S. Pollastri, M. Tattini, Flavonols: old compounds for old roles, Annals of Botany, 108 (2011) 1225-1233.

[37] R.A. Dixon, N.L. Paiva, Stress-Induced Phenylpropanoid Metabolism, Plant Cell, 7 (1995) 1085-1097.

[38] B. Winkel-Shirley, Molecular genetics and control of anthocyanin expression, in: Advances in Botanical Research, 2002, pp. 75-94.

[39] L.P. Taylor, E. Grotewold, Flavonoids as developmental regulators, Current Opinion in Plant Biology, 8 (2005) 317-323.

[40] M.R. Roberts, N.D. Paul, Seduced by the dark side: integrating molecular and ecological perspectives on the inflence of light on plant defence against pests and pathogens, New Phytologist, 170 (2006) 677-699.

[41] M.L. Falcone Ferreyra, S.P. Rius, P. Casati, Flavonoids: biosynthesis, biological functions, and biotechnological applications, Frontiers in Plant Science, 3 (2012) 1-15.

[42] S. Teng, J. Keurentjes, L. Bentsink, M. Koornneef, S. Smeekens, Sucrose-specific induction of anthocyanin biosynthesis in Arabidopsis requires the MYB75/PAP1 gene, Plant Physiology, 139 (2005) 1840-1852.

[43] U. Diaz, V. Saliba-Colombani, O. Loudet, P. Belluomo, L. Moreau, F. Daniel-Vedele, J.F. Morot-Gaudry, U. Maselaux-Daubresse, Leaf yellowing and anthocyanin accumulation are two genetically independent strategies in response to nitrogen limitation in Arabidopsis thaliana, Plant and Cell Physiology, 47 (2006) 74-83.

[44] M. Korn, T. Gaertner, A. Erban, J. Kopka, J. Selbig, D.K. Hincha, Predicting Arabidopsis Freezing Tolerance and Heterosis in Freezing Tolerance from Metabolite Composition, Molecular Plant, 3 (2010) 224-235.

[45] M. Korn, S. Peterek, H.-P. Mock, A.G. Heyer, D.K. Hincha, Heterosis in the freezing tolerance, and sugar and flavonoid contents of crosses between Arabidopsis thaliana accessions of widely varying freezing tolerance, Plant Cell and Environment, 31 (2008) 813-827.

[46] F. Matsuda, R. Nakabayashi, Y. Sawada, M. Suzuki, M.Y. Hirai, S. Kanaya, K. Saito, Mass spectra-based framework for automated structural elucidation of metabolome 
data to explore phytochemical diversity, Frontiers in Plant Science, 2 (2011) 40.

[47] S. Martens, G. Forkmann, L. Britsch, F. Wellmann, U. Matern, R. Lukacin, Divergent evolution of flavonoid 2-oxoglutarate-dependent dioxygenases in parsley, FEBS Letters, 544 (2003) 93-98.

[48] L. Caputi, M. Malnoy, V. Goremykin, S. Nikiforova, S. Martens, A genome-wide phylogenetic reconstruction of family 1 UDP-glycosyltransferases revealed the expansion of the family during the adaptation of plants to life on land, Plant Journal, 69 (2011) 1030-1042.

[49] M. Bredebach, U. Matern, S. Martens, Three 2-oxoglutarate-dependent dioxygenase activities of Equisetum arvense L. forming flavone and flavonol from (2S)-naringenin, Phytochemistry, 72 (2011) 557-563.

[50] T. Tohge, M. Watanabe, R. Hoefgen, A. Fernie, R., The evolution of phenylpropanoid metabolism in the green lineage, Critical Reviews in Biochemistry and Molecular biology, in-press (2013).

[51] J.J. Turnbull, J.-I. Nakajima, R.W.D. Welford, M. Yamazaki, K. Saito, C.J. Schofield, Mechanistic studies on three 2-oxoglutarate-dependent oxygenases of flavonoid biosynthesis: Anthocyanidin synthase, flavonol synthase, and flavanone 36-hydroxylase, Journal of Biological Chemistry, 279 (2004) 1206-1216.

[52] K. Yonekura-Sakakibara, K. Hanada, An evolutionary view of functional diversity in family 1 glycosyltransferases, Plant Journal, 66 (2011) 182-193.

[53] J.J.B. Keurentjes, J.Y. Fu, C.H.R. de Vos, A. Lommen, R.D. Hall, R.J. Bino, L.H.W. van der Plas, R.C. Jansen, D. Vreugdenhil, M. Koornneef, The genetics of plant metabolism, Nature Genetics, 38 (2006) 842-849.

[54] T. Tohge, A.R. Fernie, Combining genetic diversity, informatics and metabolomics to facilitate annotation of plant gene function., Nature Protocols, 5 (2010) 1210-1227.

[55] J.-M. Routaboul, C. Dubos, G. Beck, C. Marquis, P. Bidzinski, O. Loudet, L. Lepiniec, Metabolite profiling and quantitative genetics of natural variation for flavonoids in Arabidopsis, Journal of Experimental Botany, 63 (2012) 3749-3764.

[56] C.M. Fraser, C. Chapple, The Phenylpropanoid Pathway in Arabidopsis, The Arabidopsis Book, (2011) e0152.

[57] H. Maeda, N. Dudareva, The Shikimate Pathway and Aromatic Amino Acid Biosynthesis in Plants, Annual Review of Plant Biology, 63 (2012) 73-105.

[58] S. Ohl, S.A. Hedrick, J. Chory, C.J. Lamb, Functional properties of a phenylalanine ammonia-lyase promoter from Arabidopsis, Plant Cell, 2 (1990) 837-848.

[59] D. Shufflebottom, K. Edwards, W. Schuch, M. Bevan, Transcription of two members of a gene family encoding phenylalanine ammonia-lyase leads to remarkably different cell 
specificities and induction patterns., Plant Journal, 3 (1993) 835-845.

[60] A. Rohde, K. Morreel, J. Ralph, G. Goeminne, V. Hostyn, R. De Rycke, S. Kushnir, J. Van Doorsselaere, J.P. Joseleau, M. Vuylsteke, G. Van Driessche, J. Van Beeumen, E. Messens, W. Boerjan, Molecular phenotyping of the pal1 and pal2 mutants of Arabidopsis thaliana reveals far-reaching consequences on phenylpropanoid, amino acid, and carbohydrate metabolism., Plant Cell, 16 (2004) 2749.

[61] J. Raes, A. Rohde, J.H. Christensen, Y. Van de Peer, W. Boerjan, Genome-wide characterization of the lignification toolbox in Arabidopsis, Plant Physiology, 133 (2003) 1051-1071.

[62] F.C. Cochrane, L.B. Davin, N.G. Lewis, The Arabidopsis phenylalanine ammonia lyase gene family: kinetic characterization of the four PAL isoforms, Phytochemistry, 65 (2004) 1557-1564.

[63] J. Huang, M. Gu, Z. Lai, B. Fan, K. Shi, Y.H. Zhou, J.Q. Yu, Z. Chen, Functional analysis of the Arabidopsis PAL gene family in plant growth, development, and response to environmental stress., Plant Physiology, 153 (2010) 1526.

[64] M. Mizutani, D. Ohta, R. Sato, Isolation of a cDNA and a genomic clone encoding cinnamate 4-hydroxylase from Arabidopsis and its expression manner in planta, Plant Physiology, 113 (1997) 755-763.

[65] D. Werck-Reichhart, S. Bak, S. Paquette, Cytochrome P450, American Society of Plant Biologists., 2002.

[66] A.L. Schilmiller, J. Stout, J.-K. Weng, J. Humphreys, M.O. Ruegger, C. Chapple, Mutations in the cinnamate 4-hydroxylase gene impact metabolism, growth and development in Arabidopsis, Plant Journal, 60 (2009) 771-782.

[67] J. Ehlting, D. Büttner, Q. Wang, C.J. Douglas, I.E. Somssich, E. Kombrink, Three 4-coumarate : coenzyme A ligases in Arabidopsis thaliana represent two evolutionarily divergent classes in angiosperms., Plant Journal, 19 (1999) 9.

[68] B. Hamberger, K. Hahlbrock, The 4-coumarate : CoA ligase gene family in Arabidopsis thaliana comprises one rare, sinapate-activating and three commonly occurring isoenzymes, Proc Natl Acad Sci U S A, 101 (2004) 2209-2214.

[69] B.J. Nikolau, J.B. Ohlrogge, E.S. Wurtele, Plant biotin-containing carboxylases, Archives of Biochemistry and Biophysics, 414 (2003) 211-222.

[70] C. Alban, D. Job, R. Douce, Biotin metabolism in plants, Annual Review of Plant Physiology and Plant Molecular Biology, 51 (2000) 17-47.

[71] Y. Sasaki, Y. Nagano, Plant acetyl-CoA carboxylase: structure, biosynthesis, regulation, and gene manipulation for plant breeding, Bioscience, Biotechnology, and Biochemistry, 68 (2004) 1175-1184. 
[72] X. Li, H. Ilarslan, L. Brachova, H.R. Qian, L. Li, P. Che, E.S. Wurtele, B.J. Nikolau, Reverse-genetic analysis of the two biotin-containing subunit genes of the heteromeric acetyl-coenzyme A carboxylase in Arabidopsis indicates a unidirectional functional redundancy, Plant Physiology, 155 (2011) 293-314.

[73] K.R. Roesler, B.S. Shorrosh, J.B. Ohlrogge, Structure and expression of an Arabidopsis acetyl-coenzyme A carboxylase gene, Plant Physiology, 105 (1994) 611-617.

[74] Y. Yanai, T. Kawasaki, H. Shimada, E.S. Wurtele, B.J. Nikolau, N. Ichikawa, Genomic organization of $251 \mathrm{kDa}$ acetyl-CoA carboxylase genes in Arabidopsis: tandem gene duplication has made two differentially expressed isozymes, Plant and Cell Physiology, 36 (1995) 779-787.

[75] E. Babiychuk, K. Vandepoele, J. Wissing, M. Garcia-Diaz, R. De Rycke, H. Akbari, J. Joubes, T. Beeckman, L. Jansch, M. Frentzen, M.C.E. Van Montagu, S. Kushnir, Plastid gene expression and plant development require a plastidic protein of the mitochondrial transcription termination factor family, Proc Natl Acad Sci U S A, 108 (2011) 6674-6679.

[76] J. Puyaubert, L. Denis, C. Alban, Dual targeting of Arabidopsis holocarboxylase synthetase1: a small upstream open reading frame regulates translation initiation and protein targeting, Plant Physiology, 146 (2008) 478-491.

[77] B.L. Fatland, B.J. Nikolau, E.S. Wurtele, Reverse genetic characterization of cytosolic acetyl-CoA generation by ATP-citrate lyase in Arabidopsis, Plant Cell, 17 (2005) 182-203.

[78] S. Baud, Y. Bellec, M. Miquel, C. Bellini, M. Caboche, L. Lepiniec, J.D. Faure, C. Rochat, gurke and pasticcino3 mutants affected in embryo development are impaired in acetyl-CoA carboxylase, EMBO Reports, 5 (2004) 515-520.

[79] A. Amid, A. Lytovchenko, A.R. Fernie, G. Warren, G.J. Thorlby, The sensitive to freezing3 mutation of Arabidopsis thaliana is a cold-sensitive allele of homomeric acetyl-CoA carboxylase that results in cold-induced cuticle deficiencies, Journal of Experimental Botany, 63 (2012) 5289-5299.

[80] S.Y. Lu, H.Y. Zhao, E.P. Parsons, C.C. Xu, D.K. Kosma, X.J. Xu, D. Chao, G. Lohrey, D.K. Bangarusamy, G. Wang, R.A. Bressan, M.A. Jenks, The glossyhead1 allele of ACC1 reveals a principal role for multidomain acetyl-coenzyme A carboxylase in the biosynthesis of cuticular waxes by Arabidopsis, Plant Physiology, 157 (2011) 1079-1092.

[81] A.J. White, S.Z. Hanley, K.M. Elborough, A.R. Slabas, Physiological and biochemical consequences of down regulation, using antisense, of the high molecular weight form of acetyl CoA carboxylase in Brassica napus, in: J. Sanchez, E. Cerda-Olmedo, E. Martinez-Force (Eds.) Advances in Plant Lipid Research, Universidad de Sevilla, 
Sevilla, 1998, pp. 63-66.

[82] S. Baud, V. Guyon, J. Kronenberger, S. Wuilleme, M. Miquel, M. Caboche, L. Lepiniec, C. Rochat, Multifunctional acetyl-CoA carboxylase 1 is essential for very long chain fatty acid elongation and embryo development in Arabidopsis, Plant Journal, 33 (2003) 75-86.

[83] J. Ebel, K. Hahlbrock, Enzymes of flavone and flavonol-glycoside biosynthesis. Coordinated and selective induction in cell-suspension cultures of Petroselinum hortense, European Journal of Biochemistry, 75 (1977) 201-209.

[84] B.S. Shorrosh, R.A. Dixon, J.B. Ohlrogge, Molecular cloning, characterization, and elicitation of acetyl-CoA carboxylase from alfalfa, Proc Natl Acad Sci U S A, 91 (1994) 4323-4327.

[85] M. Kusano, T. Tohge, A. Fukushima, M. Kobayashi, N. Hayashi, H. Otsuki, Y. Kondou, H. Goto, M. Kawashima, et al., Metabolomics reveals comprehensive reprogramming involving two independent metabolic responses of Arabidopsis to UV-B light, Plant Journal, 67 (2011) 354-369.

[86] T. Konishi, T. Kamoi, R. Matsuno, Y. Sasaki, Induction of cytosolic acetyl-coenzyme A carboxylase in pea leaves by ultraviolet-B irradiation, Plant and Cell Physiology, 37 (1996) 1197-1200.

[87] M.B. Austin, J.P. Noel, The chalcone synthase superfamily of type III polyketide synthases, Natural product reports, 20 (2003) 79-110.

[88] B.W. Shirley, W.L. Kubasek, G. Storz, E. Bruggemann, M. Koornneef, F.M. Ausubel, H.M. Goodman, Analysis of Arabidopsis Mutants Deficient in Flavonoid Biosynthesis, Plant Journal, 8 (1995) 659-671.

[89] S.S. Kim, E. Grienenberger, B. Lallemand, C.C. Colpitts, S.Y. Kim, A. Souza Cde, P. Geoffroy, D. Heintz, D. Krahn, M. Kaiser, E. Kombrink, T. Heitz, D.Y. Suh, M. Legrand, C.J. Douglas, LAP6/POLYKETIDE SYNTHASE A and LAP5/POLYKETIDE SYNTHASE B encode hydroxyalkyl alpha-pyrone synthases required for pollen development and sporopollenin biosynthesis in Arabidopsis thaliana, Plant Cell, 22 (2010) 4045-4066.

[90] A.A. Dobritsa, Z. Lei, S. Nishikawa, E. Urbanczyk-Wochniak, D.V. Huhman, D. Preuss, L.W. Sumner, LAP5 and LAP6 encode anther-specific proteins with similarity to chalcone synthase essential for pollen exine development in Arabidopsis, Plant Physiology, 153 (2010) 937-955.

[91] M.N. Ngaki, G.V. Louie, R.N. Philippe, G. Manning, F. Pojer, M.E. Bowman, L. Li, E. Larsen, E.S. Wurtele, J.P. Noel, Evolution of the chalcone-isomerase fold from fatty-acid binding to stereospecific catalysis, Nature, 485 (2012) 530-533. 
[92] M.K. Pelletier, B.W. Shirley, Analysis of flavanone 3-hydroxylase in Arabidopsis seedlings - Coordinate regulation with chalcone synthase and chalcone isomerase, Plant Physiology, 111 (1996) 339-345.

[93] D.K. Owens, K.C. Crosby, J. Runac, B.A. Howard, B.S.J. Winkel, Biochemical and genetic characterization of Arabidopsis flavanone 3b-hydroxylase, Plant Physiology and Biochemistry, 46 (2008) 833-843.

[94] C. Schoenbohm, S. Martens, C. Eder, G. Forkmann, B. Weisshaar, Identification of the Arabidopsis thaliana flavonoid 3'-hydroxylase gene and functional expression of the encoded P450 enzyme, Biological Chemistry, 381 (2000) 749-753.

[95] M.K. Pelletier, J.R. Murrell, B.W. Shirley, Characterization of flavonol synthase and leucoanthocyanidin dioxygenase genes in Arabidopsis - Further evidence for differential regulation of "early" and "late" genes, Plant Physiology, 113 (1997) 1437-1445.

[96] A.G. Prescott, N.P.J. Stamford, G. Wheeler, J.L. Firmin, In vitro properties of a recombinant flavonol synthase from Arabidopsis thaliana, Phytochemistry, 60 (2002) 589-593.

[97] E. Wisman, U. Hartmann, M. Sagasser, E. Baumann, K. Palme, K. Hahlbrock, H. Saedler, B. Weisshaar, Knock-out mutants from an En-1 mutagenized Arabidopsis thaliana population generate phenylpropanoid biosynthesis phenotypes, Proc Natl Acad Sci U S A, 95 (1998) 12432-12437.

[98] D.K. Owens, A.B. Alerding, K.C. Crosby, A.B. Bandara, J.H. Westwood, B.S.J. Winkel, Functional Analysis of a Predicted Flavonol Synthase Gene Family in Arabidopsis, Plant Physiology, 147 (2008) 1046-1061.

[99] R. Stracke, R. De Vos, L. Bartelniewoehner, H. Ishihara, M. Sagasser, S. Martens, B. Weisshaar, Metabolomic and genetic analyses of flavonol synthesis in \&lt;i\&gt;Arabidopsis thaliana support the in vivo involvement of leucoanthocyanidin dioxygenase, Planta, 229 (2009) 427-445.

[100] A. Preuß, R. Stracke, B. Weisshaar, A. Hillebrecht, U. Matern, S. Martens, Arabidopsis thaliana expresses a second functional flavonol synthase, FEBS Letters, 583 (2009) 1981-1986.

[101] B.W. Shirley, S. Hanley, H.M. Goodman, Effects of Ionizing-Radiation on a Plant Genome - Analysis of 2 Arabidopsis Transparent-Testa Mutations, Plant Cell, 4 (1992) 333-347.

[102] P. Bowerman, M. Ramirez, M. Moore, R. Helm, B. Winkel, Analysis of T-DNA alleles of flavonoid biosynthesis genes in Arabidopsis ecotype Columbia, BMC Research Notes, 5 (2012) 485. 
[103] S. Abrahams, E. Lee, A.R. Walker, G.J. Tanner, P.J. Larkin, A.R. Ashton, The Arabidopsis TDS4 gene encodes leucoanthocyanidin dioxygenase (LDOX) and is essential for proanthocyanidin synthesis and vacuole development, Plant Journal, 35 (2003) 624-636.

[104] J.-I. Nakajima, Y. Tanaka, M. Yamazaki, K. Saito, Reaction Mechanism from Leucoanthocyanidin to Anthocyanidin 3-Glucoside, a Key Reaction for Coloring in Anthocyanin Biosynthesis, Journal of Biological Chemistry, 276 (2001) 25797-25803.

[105] R.W. Welford, J.J. Turnbull, T.D. Claridge, A.G. Prescott, C.J. Schofield, Evidence for oxidation at C-3 of the flavonoid C-ring during anthocyanin biosynthesis, Chem Commun (Camb), (2001) 1828-1829.

[106] K. Saito, M. Kobayashi, Z. Gong, Y. Tanaka, M. Yamazaki, Direct evidence for anthocyanidin synthase as a 2-oxoglutarate-dependent oxygenase: molecular cloning and functional expression of cDNA from a red forma of Perilla frutescens, Plant Journal, 17 (1999) 181-189.

[107] S. Martens, A. Preuß, U. Matern, Multifunctional flavonoid dioxygenases: Flavonol and anthocyanin biosynthesis in Arabidopsis thaliana L, Phytochemistry, 71 (2010) 1040-1049.

[108] J.-i. Nakajima, Y. Sato, T. Hoshino, M. Yamazaki, K. Saito, Mechanistic Study on the Oxidation of Anthocyanidin Synthase by Quantum Mechanical Calculation, Journal of Biological Chemistry, 281 (2006) 21387-21398.

[109] M. Devic, J. Guilleminot, I. Debeaujon, N. Bechtold, E. Bensaude, M. Koornneef, G. Pelletier, M. Delseny, The BANYULS gene encodes a DFR-like protein and is a marker of early seed coat development, Plant Journal, 19 (1999) 387-398.

[110] D.-Y. Xie, S.B. Sharma, R.A. Dixon, Anthocyanidin reductases from Medicago truncatula and Arabidopsis thaliana, Archives of Biochemistry and Biophysics, 422 (2004) 91-102.

[111] D.Y. Xie, S.B. Sharma, N.L. Paiva, D. Ferreira, R.A. Dixon, Role of anthocyanidin reductase, encoded by BANYULS in plant flavonoid biosynthesis, Science., 299 (2003) 396-399.

[112] J. Bogs, M.O. Downey, J.S. Harvey, A.R. Ashton, G.J. Tanner, S.P. Robinson, Proanthocyanidin Synthesis and Expression of Genes Encoding Leucoanthocyanidin Reductase and Anthocyanidin Reductase in Developing Grape Berries and Grapevine Leaves, Plant Physiology, 139 (2005) 652-663.

[113] L. Yuan, L. Wang, Z. Han, Y. Jiang, L. Zhao, H. Liu, L. Yang, K. Luo, Molecular cloning and characterization of PtrLAR3, a gene encoding leucoanthocyanidin reductase from Populus trichocarpa, and its constitutive expression enhances fungal resistance in 
transgenic plants, Journal of Experimental Botany, 63 (2012) 2513-2524.

[114] L. Pourcel, J.M. Routaboul, L. Kerhoas, M. Caboche, L. Lepiniec, I. Debeaujon, TRANSPARENT TESTA10 encodes a laccase-like enzyme involved in oxidative polymerization of flavonoids in Arabidopsis seed coat, Plant Cell, 17 (2005) 2966-2980.

[115] L. Pourcel, J.-M. Routaboul, V. Cheynier, L. Lepiniec, I. Debeaujon, Flavonoid oxidation in plants: from biochemical properties to physiological functions, Trends in Plant Science, 12 (2007) 29-36.

[116] Y. Li, S. Baldauf, E.K. Lim, D.J. Bowles, Phylogenetic analysis of the UDP-glycosyltransferase multigene family of Arabidopsis thaliana, Journal of Biological Chemistry, 276 (2001) 4338-4343.

[117] J.C. D'Auria, J. Gershenzon, The secondary metabolism of Arabidopsis thaliana: growing like a weed, Current Opinion in Plant Biology, 8 (2005) 308-316.

[118] P. Jones, B. Messner, J. Nakajima, A.R. Schaffner, K. Saito, UGT73C6 and UGT78D1, glycosyltransferases involved in flavonol glycoside biosynthesis in Arabidopsis thaliana, Journal of Biological Chemistry, 278 (2003) 43910-43918.

[119] K. Yonekura-Sakakibara, T. Tohge, R. Niida, K. Saito, Identification of a flavonol 7-O-rhamnosyltransferase gene determining flavonoid pattern in Arabidopsis by transcriptome coexpression analysis and reverse genetics, Journal of Biological Chemistry, 282 (2007) 14932-14941.

[120] K. Yonekura-Sakakibara, A. Fukushima, R. Nakabayashi, K. Hanada, F. Matsuda, S. Sugawara, E. Inoue, T. Kuromori, T. Ito, et al., Two glycosyltransferases involved in anthocyanin modification delineated by transcriptome independent component analysis in Arabidopsis thaliana, Plant Journal, 69 (2012) 154-167.

[121] Y. Matsuba, N. Sasaki, M. Tera, M. Okamura, Y. Abe, E. Okamoto, H. Nakamura, H. Funabashi, M. Takatsu, M. Saito, H. Matsuoka, K. Nagasawa, Y. Ozeki, A novel glucosylation reaction on anthocyanins catalyzed by acyl-glucose-dependent glucosyltransferase in the petals of carnation and delphinium, Plant Cell, 22 (2010) 3374-3389.

[122] T. Miyahara, M. Takahashi, Y. Ozeki, N. Sasaki, Isolation of an acyl-glucose-dependent anthocyanin 7-O-glucosyltransferase from the monocot Agapanthus africanus, Journal of Plant Physiology, 169 (2012) 1321-1326.

[123] T. Miyahara, R. Sakiyama, Y. Ozeki, N. Sasaki, Acyl-glucose-dependent glucosyltransferase catalyzes the final step of anthocyanin formation in Arabidopsis, Journal of Plant Physiology, (2013) in press.

[124] K. Yonekura-Sakakibara, T. Nakayama, M. Yamazaki, K. Saito, Modification and stabilization of anthocyanins, in: K. Gould, K. Davies, C. Winefield (Eds.) Anthocyanins. 
Biosynthesis, Functions, and Applications, Springer, New York, 2008, pp. 169-190.

[125] J.C. D'Auria, M. Reichelt, K. Luck, A. Svatos, J. Gershenzon, Identification and characterization of the BAHD acyltransferase malonyl CoA: Anthocyanidin 5-O-glucoside-6 "-O-malonyltransferase (At5MAT) in Arabidopsis thaliana, FEBS Letters, 581 (2007) 872-878.

[126] I. Muzac, J. Wang, D. Anzellotti, H. Zhang, R.K. Ibrahim, Functional expression of an Arabidopsis cDNA clone encoding a flavonol 3'-O-methyltransferase and characterization of the gene product, Archives of Biochemistry and Biophysics, 375 (2000) 385-388.

[127] T. Goujon, R. Sibout, B. Pollet, B. Maba, L. Nussaume, N. Bechtold, F. Lu, J. Ralph, I. Mila, Y. Barrière, C. Lapierre, L. Jouanin, A new Arabidopsis thaliana mutant deficient in the expression of O-methyltransferase impacts lignins and sinapoyl esters, Plant Molecular Biology, 51 (2003) 973-989.

[128] B. St-Pierre, V.D. Luca, Chapter Nine Evolution of acyltransferase genes: Origin and diversification fo the BAHD superfamily of acyltransferases involved in secondary metabolism, in: R.I.L.V. John T. Romeo, L. Vincenzo De (Eds.) Recent Advances in Phytochemistry, Elsevier, 2000, pp. 285-315.

[129] C. Milkowski, D. Strack, Serine carboxypeptidase-like acyltransferases, Phytochemistry, 65 (2004) 517-524.

[130] J. Luo, Y. Nishiyama, C. Fuell, G. Taguchi, K. Elliott, L. Hill, Y. Tanaka, M. Kitayama, M. Yamazaki, P. Bailey, A. Parr, A.J. Michael, K. Saito, C. Martin, Convergent evolution in the BAHD family of acyl transferases: identification and characterization of anthocyanin acyl transferases from Arabidopsis thaliana, Plant Journal, 50 (2007) 678-695.

[131] C.M. Fraser, M.G. Thompson, A.M. Shirley, J. Ralph, J.A. Schoenherr, T. Sinlapadech, M.C. Hall, C. Chapple, Related Arabidopsis serine carboxypeptidase-like sinapoylglucose acyltransferases display distinct but overlapping substrate specificities, Plant Physiology, 144 (2007) 1986-1999.

[132] H. Kubo, N. Nawa, S.A. Lupsea, Anthocyaninless1 gene of Arabidopsis thaliana encodes a UDP-glucose:flavonoid-3-O-glucosyltransferase, Journal of Plant Research, 120 (2007) 445-449.

[133] M. Yamazaki, E. Yamagishi, Z. Gong, M. Fukuchi-Mizutani, Y. Fukui, Y. Tanaka, T. Kusumi, M. Yamaguchi, K. Saito, Two flavonoid glucosyltransferases from Petunia hybrida: molecular cloning, biochemical properties and developmentally regulated expression, Plant Molecular Biology, 48 (2002) 401-411.

[134] M. Yamazaki, Z. Gong, M. Fukuchi-Mizutani, Y. Fukui, Y. Tanaka, T. Kusumi, K. Saito, 
Molecular cloning and biochemical characterization of a novel anthocyanin 5-O-glucosyltransferase by mRNA differential display for plant forms regarding anthocyanin, Journal of Biological Chemistry, 274 (1999) 7405-7411.

[135] E.K. Lim, Y. Li, A. Parr, R. Jackson, D.A. Ashford, D.J. Bowles, Identification of glucosyltransferase genes involved in sinapate metabolism and lignin synthesis in Arabidopsis, Journal of Biological Chemistry, 276 (2001) 4344-4349.

[136] C.M. Fraser, M.G. Thompson, A.M. Shirley, J. Ralph, J.A. Schoenherr, T. Sinlapadech, M.C. Hall, C. Chapple, Related Arabidopsis serine carboxypeptidase - Like sinapoylglucose acyltransferases display distinct but overlapping substrate Specificities, Plant Physiology, 144 (2007) 1986-1999.

[137] Y. Morita, A. Hoshino, Y. Kikuchi, H. Okuhara, E. Ono, Y. Tanaka, Y. Fukui, N. Saito, E. Nitasaka, H. Noguchi, S. Iida, Japanese morning glory dusky mutants displaying reddish-brown or purplish-gray flowers are deficient in a novel glycosylation enzyme for anthocyanin biosynthesis, UDP-glucose:anthocyanidin 3-O-glucoside-2"-O-glucosyltransferase, due to 4-bp insertions in the gene, Plant Journal, 42 (2005) 353-363.

[138] S. Sawada, H. Suzuki, F. Ichimaida, M.A. Yamaguchi, T. Iwashita, Y. Fukui, H. Hemmi, T. Nishino, T. Nakayama, UDP-glucuronic acid:anthocyanin glucuronosyltransferase from red daisy (Bellis perennis) flowers. Enzymology and phylogenetics of a novel glucuronosyltransferase involved in flower pigment biosynthesis, Journal of Biological Chemistry, 280 (2005) 899-906.

[139] B. Usadel, A.M. Kuschinsky, M.G. Rosso, N. Eckermann, M. Pauly, RHM2 is involved in mucilage pectin synthesis and is required for the development of the seed coat in Arabidopsis, Plant Physiology, 134 (2004) 286-295.

[140] T.L. Western, D.S. Young, G.H. Dean, W.L. Tan, A.L. Samuels, G.W. Haughn, MUCILAGE-MODIFIED4 encodes a putative pectin biosynthetic enzyme developmentally regulated by APETALA2, TRANSPARENT TESTA GLABRA1, and GLABRA2 in the Arabidopsis seed coat, Plant Physiology, 134 (2004) 296-306.

[141] T. Oka, T. Nemoto, Y. Jigami, Functional analysis of Arabidopsis thaliana RHM2/MUM4, a multidomain protein involved in UDP-D-glucose to UDP-L-rhamnose conversion, Journal of Biological Chemistry, 282 (2007) 5389-5403.

[142] N. Shikazono, A. Tanaka, H. Watanabe, S. Tano, Rearrangements of the DNA in carbon ion-induced mutants of Arabidopsis thaliana, Genetics, 157 (2001) 379-387.

[143] S. Kitamura, N. Shikazono, A. Tanaka, TRANSPARENT TESTA 19 is involved in the accumulation of both anthocyanins and proanthocyanidins in Arabidopsis, Plant Journal, 37 (2004) 104-114. 
[144] J. Zhao, Y. Pang, R.A. Dixon, The Mysteries of Proanthocyanidin Transport and Polymerization, Plant Physiology, 153 (2010) 437-443.

[145] I. Debeaujon, A.J.M. Peeters, K.M. Leon-Kloosterziel, M. Koornneef, The TRANSPARENT TESTA12 gene of Arabidopsis encodes a multidrug secondary transporter-like protein required for flavonoid sequestration in vacuoles of the seed coat endothelium, Plant Cell, 13 (2001) 853-871.

[146] K. Marinova, L. Pourcel, B. Weder, M. Schwarz, D. Barron, J.M. Routaboul, I. Debeaujon, M. Klein, The Arabidopsis MATE transporter TT12 acts as a vacuolar flavonoid/H+-antiporter active in proanthocyanidin-accumulating cells of the seed coat, Plant Cell, 19 (2007) 2023-2038.

[147] E.P. Thompson, C. Wilkins, V. Demidchik, J.M. Davies, B.J. Glover, An Arabidopsis flavonoid transporter is required for anther dehiscence and pollen development, Journal of Experimental Botany, 61 (2010) 439-451.

[148] K.A. Marrs, M.R. Alfenito, A.M. Lloyd, V. Walbot, A Glutathione-S-Transferase Involved in Vacuolar Transfer Encoded by the Maize Gene Bronze-2, Nature, 375 (1995) 397-400.

[149] B. Wangwattana, Y. Koyama, Y. Nishiyama, M. Kitayama, M. Yamazaki, K. Saito, Characterization of PAP1-upregulated Glutathione S-transferase genes in Arabidopsis thaliana, Plant Biotechnology, 25 (2008) 191-196.

[150] M.R. Alfenito, E. Souer, C.D. Goodman, R. Buell, J. Mol, R. Koes, V. Walbot, Functional complementation of anthocyanin sequestration in the vacuole by widely divergent glutathione S-transferases, Plant Cell, 10 (1998) 1135-1149.

[151] L. Loyall, K. Uchida, S. Braun, M. Furuya, H. Frohnmeyer, Glutathione and a UV light-induced glutathione S-transferase are involved in signaling to chalcone synthase in cell cultures, Plant Cell, 12 (2000) 1939-1950.

[152] L.A. Mueller, C.D. Goodman, R.A. Silady, V. Walbot, AN9, a petunia glutathione S-transferase required for anthocyanin sequestration, is a flavonoid-binding protein, Plant Physiology, 123 (2000) 1561-1570.

[153] S. Kitamura, Y. Akita, H. Ishizaka, I. Narumi, A. Tanaka, Molecular characterization of an anthocyanin-related glutathione S-transferase gene in cyclamen, Journal of Plant Physiology, 169 (2012) 636-642.

[154] S. Kitamura, Transport of flavonoids, in: E. Grotewold (Ed.) Science of Flavonoids, 2008, pp. 123-146.

[155] A.P. Smith, S.D. Nourizadeh, W.A. Peer, J.H. Xu, A. Bandyopadhyay, A.S. Murphy, P.B. Goldsbrough, Arabidopsis AtGSTF2 is regulated by ethylene and auxin, and encodes a glutathione S-transferase that interacts with flavonoids, Plant Journal, 36 (2003) 
433-442.

[156] J. Zhao, R.A. Dixon, MATE Transporters Facilitate Vacuolar Uptake of Epicatechin 3 '-O-Glucoside for Proanthocyanidin Biosynthesis in Medicago truncatula and Arabidopsis, Plant Cell, 21 (2009) 2323-2340.

[157] Y. Sun, H. Li, J.-R. Huang, Arabidopsis TT19 Functions as a Carrier to Transport Anthocyanin from the Cytosol to Tonoplasts, Molecular Plant, 5 (2012) 387-400.

[158] I.R. Baxter, J.C. Young, G. Armstrong, N. Foster, N. Bogenschutz, T. Cordova, W.A. Peer, S.P. Hazen, A.S. Murphy, J.F. Harper, A plasma membrane H+-ATPase is required for the formation of proanthocyanidins in the seed coat endothelium of Arabidopsis thaliana, Proc Natl Acad Sci U S A, 102 (2005) 2649-2654.

[159] K. Yonekura-Sakakibara, A. Fukushima, K. Saito, Transcriptome data modeling toward plant metabolic engineering, Current Opinion in Biotechnology, (2013).

[160] R.A. Dixon, C. Liu, J.H. Jun, Metabolic engineering of anthocyanins and condensed tannins in plants, Current Opinion in Biotechnology, (2012) in press.

[161] A. Bar-Even, D. Salah Tawfik, Engineering specialized metabolic pathways-is there a room for enzyme improvements?, Current Opinion in Biotechnology, (2012) in press.

[162] M. Yamazaki, K. Saito, Molecular genetic study on the anthocyanin chemotypes of Perilla frutescens var. crispa, Natural product reports, 6 (2011) 423-427.

[163] K. Saito, M. Yamazaki, Biochemistry and molecular biology of the late-stage of biosynthesis of anthocyanin: lessons from Perilla frutescens as a model plant, New Phytologist, 155 (2002) 9-23.

[164] K. Yonekura-Sakakibara, K. Saito, Functional genomics for plant natural product biosynthesis, Natural product reports, 26 (2009) 1466-1487.

[165] K. Yonekura-Sakakibara, K. Saito, Review: genetically modified plants for the promotion of human health, Biotechnology Letters, 28 (2006) 1983-1991.

[166] E. Butelli, L. Titta, M. Giorgio, H.-P. Mock, A. Matros, S. Peterek, E.G.W.M. Schijlen, R.D. Hall, A.G. Bovy, J. Luo, C. Martin, Enrichment of tomato fruit with health-promoting anthocyanins by expression of select transcription factors, Nature Biotechnology, 26 (2008) 1301-1308.

[167] R. Nakabayashi, K. Saito, Metabolomics for Unknown Plant Metabolites, Analytical and Bioanalytical Chemistry, (2013) submitted.

[168] A. Oikawa, K. Saito, Metabolite analyses of single cells, Plant Journal, 70 (2012) 30-38.

[169] V. De Luca, V. Salim, S.M. Atsumi, F. Yu, Mining the biodiversity of plants: a revolution in the making, Science., 336 (2012) 1658-1661. 


\section{Anthocyanins (A1 - A11)}

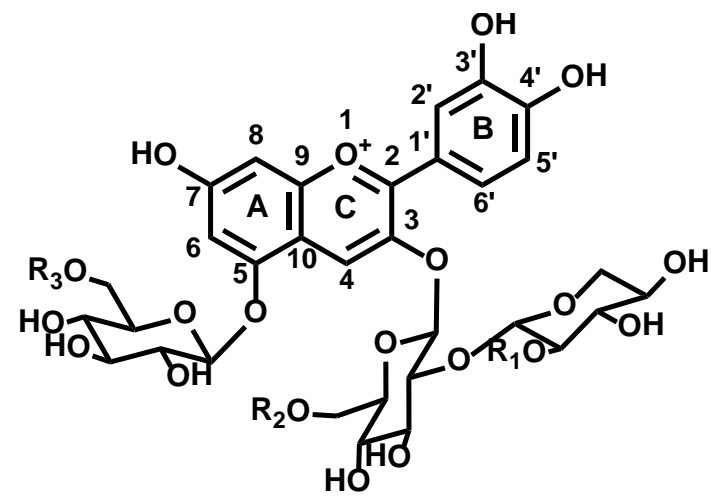

\section{Flavonols (f1 - f35)}<smiles>[R]Oc1cc(O)c2c(=O)c(O[R2])c(-c3ccc(O)c([R1])c3)oc2c1</smiles>

Proanthocyanidins (p1 - p8)<smiles>Oc1cc(O)c2c(c1)OC(c1ccc(O)c(O)c1)C(O)C2c1c(O)cc(O)c2c1OC(c1ccc(O)c(O)c1)C(O)C2c1c(O)cc(O)c2c1OC(c1ccc(O)c(O)c1)C(O)C2</smiles>

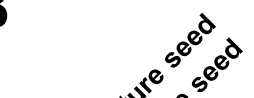

\section{.}

A1

A2

A3

A4

A5

A6

A7

A8

A9

A10

A11

f1

f2

f3

f4

f5

f6

f7

f8

f9

f10

f11

f12

f13

f14

f15

f16

f17

f18

f19

f20

f21

f22

f23

f24

f25

f26

f27

f28

f29

f30

f31

f32

f33

f34

f35

p1

p2

p3

p4

p5

p6

p7

p8
\& $\beta^{2} \quad \beta^{3}$

C $\mathrm{H} H \mathrm{H}$

$\begin{array}{llll}\mathrm{C} & \mathrm{H} & \mathrm{H} & \mathrm{mal}\end{array}$

C $\mathrm{H} p$-cou $\mathrm{H}$

$C$ sina $\mathrm{H} H$

C $\mathrm{H}$ p-cou mal

C $\mathrm{H}$ p-cou-glc $\mathrm{H}$

$C$ sina $p$-cou $H$

C $\mathrm{H}$ p-cou-glc mal

C sina $p$-cou mal

$C$ sina $p$-cou-glc $\mathrm{H}$

$C$ sina $p$-cou-glc mal

$\mathrm{K} H$ rha rha

$\mathrm{K}$ H glc rha

$\mathrm{K} \quad \mathrm{H}$ rha $(1 \rightarrow 2) \mathrm{glc}$ rha

K H glc $(1 \rightarrow 6)$ glc rha

$Q \mathrm{OH}$ rha rha

Q $\mathrm{OH}$ glc rha

$Q$ OH rha glc

$\mathrm{Q}$ OH rha $(1 \rightarrow 2)$ glc rha

$\mathrm{K} H$ conjugate

K H hex+deoxyhex -

$\mathrm{K} H$ conjugate

$\mathrm{K}$ H rha+conjugate -

$\mathrm{Q}$ OH hex+deoxyhex -

$\mathrm{OCH}_{3}$ glc rha

$\mathrm{OCH}_{3}$ rha glc

$\mathrm{Q} \mathrm{OH}$ glc $\mathrm{H}$

K H rha H

K H glc $\quad H$

$\mathrm{K} H$ ara rha

K H rha glc

K H glcthex -

$\mathrm{K} H$ pent+deoxyhex rha

$Q \mathrm{OH}$ ara $\mathrm{H}$

Q OH rha H

Q $\mathrm{OH}$ ara rha

$\mathrm{Q}$ OH glc+hex -

I $\mathrm{OCH}_{3}$ rha $\mathrm{H}$

$\mathrm{OCH}_{3}$ glc $\mathrm{H}$

$\mathrm{OCH}_{3}$ ara rha

$\mathrm{OCH}_{3}$ rha rha

$\mathrm{OCH}_{3}$ gluthex -

$\mathrm{OCH}_{3}$ rha $(1 \rightarrow 2)$ glc rha

$\mathrm{H} \quad$ rha + hex +mal

$\mathrm{OH} \quad \mathrm{rha}+$ hex +mal

$\mathrm{OCH}_{3} \quad$ rha + hex +mal

epicatechin

procyanidin $B 2(n=0)$

epicatechin trimer $(n=1)$

epicatechin tetramer $(n=2)$

epicatechin pentamer $(n=3)$

epicatechin hexamer $(n=4)$

epicatechin heptamer $(n=5)$

epicatechin hexoside 


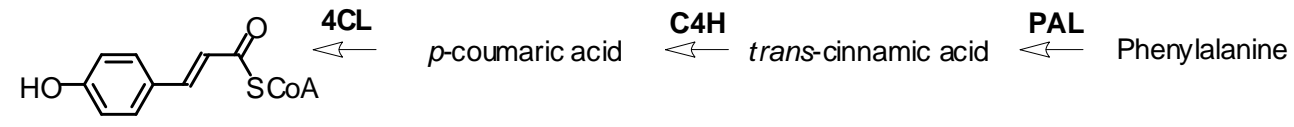

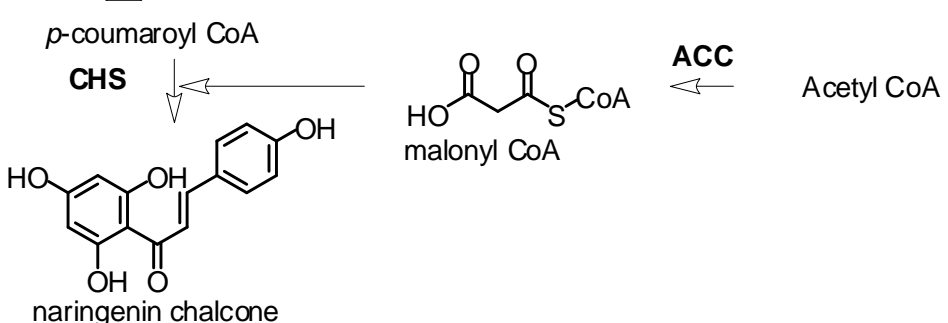

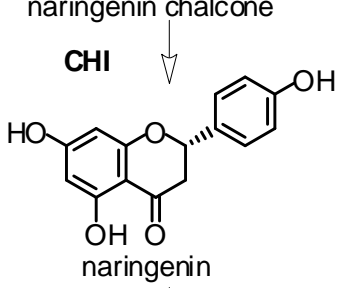<smiles>O=C1c2c(O)cc(O)cc2O[C@H](c2ccc(O)cc2)C1O</smiles>

dihydrokaempferol<smiles>O=C1c2c(O)cc(O)cc2O[C@H](c2ccc(O)c(O)c2)C1O</smiles>
dihydroquercetin

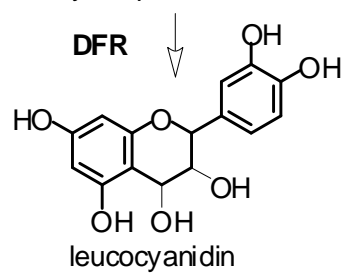<smiles></smiles><smiles>[R]NCC(C)(C)C</smiles>

(-)-epicatechin<smiles>Cc1c(O)cc(O)c2c1O[C@H](c1ccc(O)c(O)c1)[C@H](O)C2c1c(O)cc(O)c2c1O[C@H](c1ccc(O)c(O)c1)[C@H](O)C2</smiles>

oxidized procyanidin derivatives 
Fig. 3

F3AraT

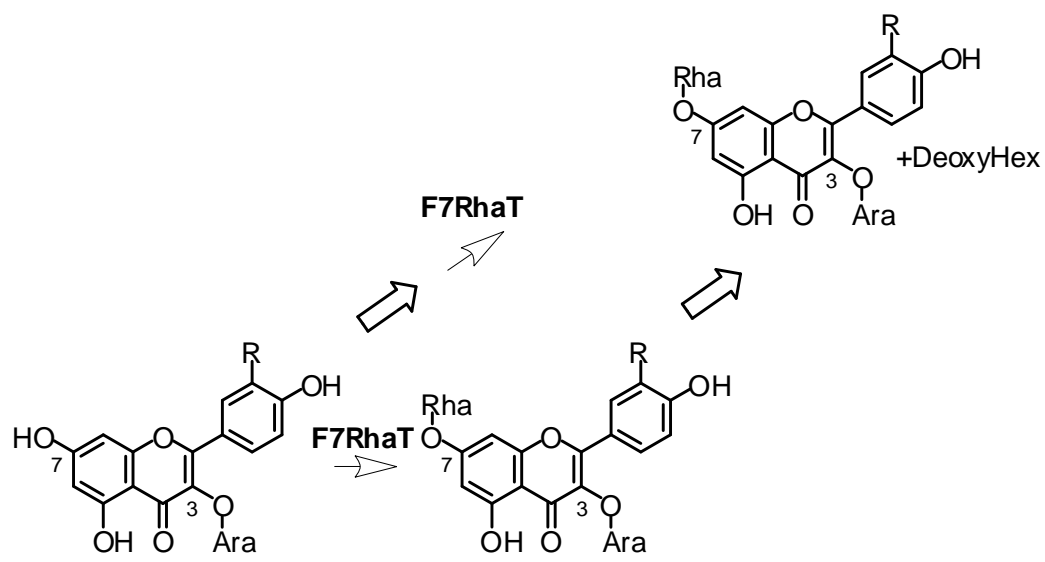

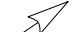<smiles>O=c1c(O)c(-c2ccc(O)cc2)oc2cc(O)cc(O)c12</smiles><smiles>O=c1c(O)c(-c2ccc(O)c(O)c2)oc2cc(O)cc(O)c12</smiles><smiles>COc1cc(-c2oc3cc(O)cc(O)c3c(=O)c2O)ccc1O</smiles>
isorhamnetin<smiles></smiles><smiles>[R]c1cc(-c2oc3cc(O)cc(O)c3c(=O)c2O[CH])ccc1O</smiles>

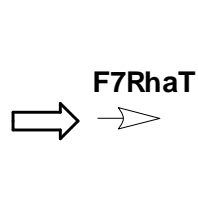<smiles></smiles>

F7RhaT<smiles></smiles><smiles>C1=CC1</smiles><smiles>[R]Oc1cc(O)c2c(=O)c(OCl)c(-c3ccc(O)c([R])c3)oc2c1</smiles>

F3RhaT<smiles>[R10]Oc1c(-c2ccc(O)c([R])c2)oc2cc(O)cc(O)c2c1=O</smiles>

F7RhaT<smiles>[R10]Oc1cc(O)c2c(=O)c(O[R])c(-c3ccc(O)c([R])c3)oc2c1</smiles> 
Fig. 4

A

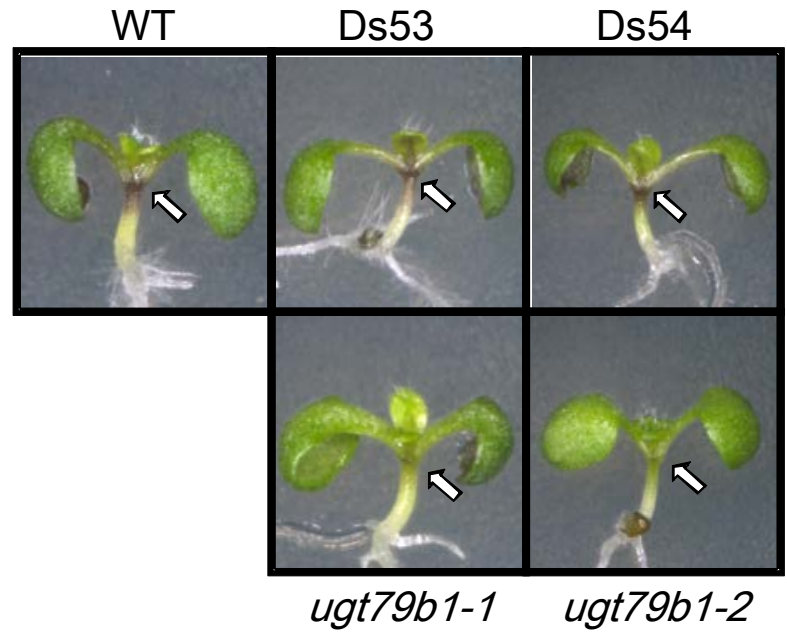

B

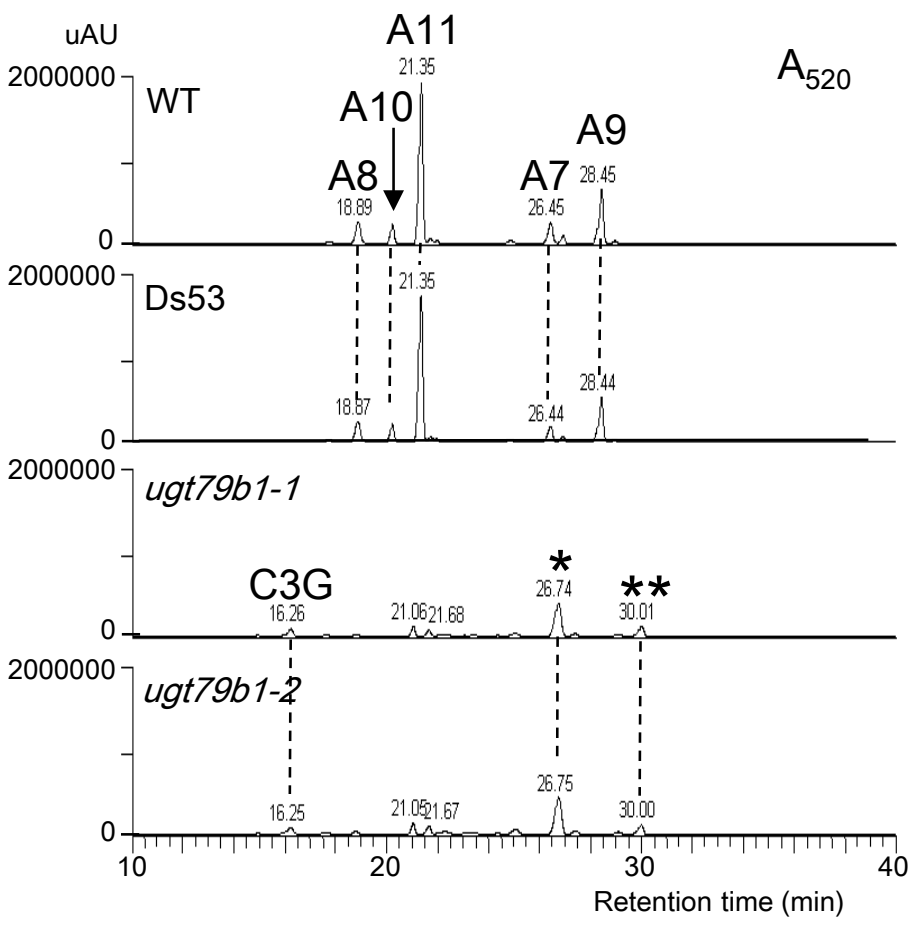


Fig. 5
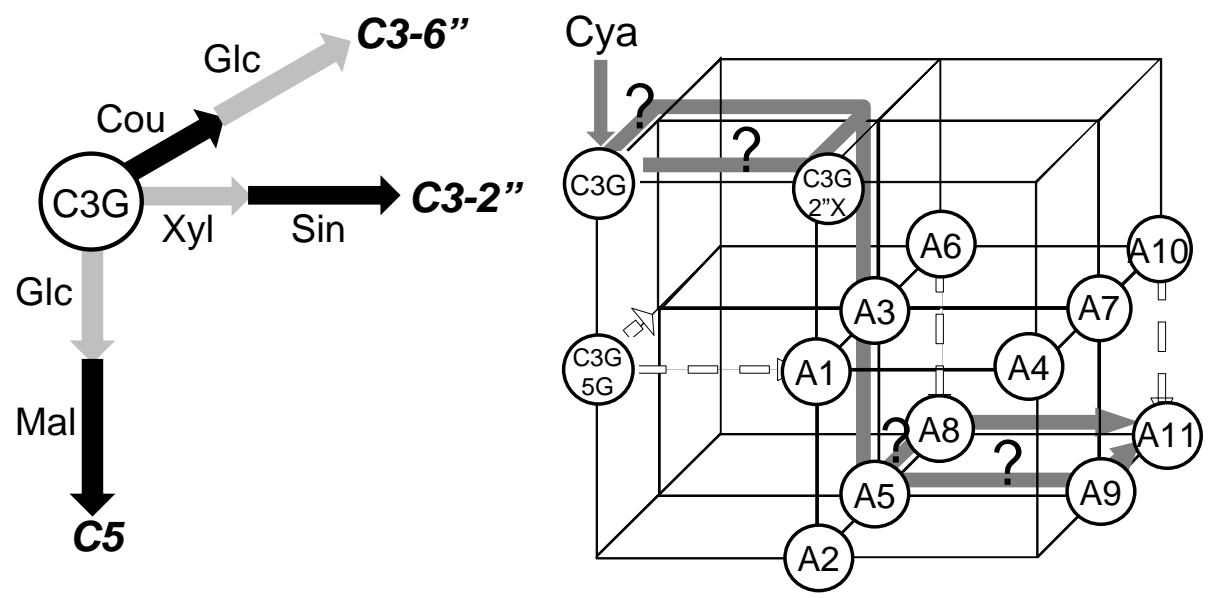